This item was submitted to Loughborough's Research Repository by the author.

Items in Figshare are protected by copyright, with all rights reserved, unless otherwise indicated.

\title{
Combined active suspension and structural damping control for suppression of flexible bodied railway vehicle vibration
}

PLEASE CITE THE PUBLISHED VERSION

https://doi.org/10.1080/00423114.2019.1572902

\section{PUBLISHER}

(C) Taylor \& Francis

\section{VERSION}

AM (Accepted Manuscript)

\section{PUBLISHER STATEMENT}

This is an Accepted Manuscript of an article published by Taylor \& Francis in Vehicle System Dynamics on 4th February 2019, available online: https://doi.org/10.1080/00423114.2019.1572902

\section{LICENCE}

CC BY-NC-ND 4.0

\section{REPOSITORY RECORD}

Zheng, Xiang, Argyrios C. Zolotas, and Roger Goodall. 2019. "Combined Active Suspension and Structural Damping Control for Suppression of Flexible Bodied Railway Vehicle Vibration”. figshare.

https://hdl.handle.net/2134/37633. 


\title{
Combined active suspension and structural damping control for suppression of flexible bodied railway vehicle vibration
}

\author{
X. Zheng*† A.C. Zolotas ${ }^{\ddagger}$ and R.M.Goodall ${ }^{\S}$
}

\begin{abstract}
The design trend for future high speed trains is envisaged to be lightweight, rising the cost of structural vibration due to the extra flexibility. In this context, studies have looked into suppression of such vibrations via use of either (conventional actuators) active suspensions or by structural damping via piezoelectric actuators. The addition of extra macro-actuators will highly impact vehicle weight and is subject to location constraints, while the use of only piezoactuators normally does not reach the required force levels for appropriate suppression. However, piezo-actuators provide appropriate complementary action with conventional active suspension. In this paper, we present a decentralised control scheme for suppressing the vertical vibration of the vehicle body, combining active structural damping via frequency-weighted $\mathcal{H}_{2}$ control and active suspension control using skyhook damping via structured $\mathcal{H}_{\infty}$ synthesis. A vertical side-view model of a flexible-bodied railway vehicle is used for the control study, with the configuration of piezoelectric actuators and sensors determined via structural norms. Stability robustness of the controller is analysed with respect to parametric and dynamic uncertainties using $\mu$ analysis techniques. Results illustrate the effectiveness of the proposed control scheme for both flexible and rigid modes while guaranteeing robustness to model uncertainty.
\end{abstract}

Keywords: railway vehicle; active structural damping; active suspension control; frequency-weighted $\mathcal{H}_{2}$ control; skyhook damping; $\mu$ analysis

\section{Introduction}

Future railway vehicles tend to be designed lighter to achieve higher speed and also to address cost-effectiveness and energy-efficiency. However, lightweight vehicle bodies

\footnotetext{
${ }^{*}$ Corresponding author

${ }^{\dagger}$ Key Lab of Marine Technology and Control Engineering, Institute of Logistics Science and Engineering,Shanghai Maritime University, Shanghai 201306, P. R. China,xiangzheng@shmtu.edu.cn

${ }^{\ddagger}$ Centre for Autonomous and Cyber-Physical Systems, Cranfield University, Cranfield, Bedfordshire, UK argyrios.zolotas@cranfield.ac.uk

${ }^{\S}$ Control Systems Group, Department of Electronic and Electrical Engineering, Loughborough University, Loughborough, Leicestershire LE11 3TU,UK, r.m.goodall@lboro.ac.uk
} 
are more flexible with structural vibration easily excited by exogenous disturbances, resulting in increased levels of high-frequency vibration. Therefore, suppression of vibration of flexible modes as well as the rigid modes of the vehicle body is important in order to provide appropriate passenger ride quality.

Vibration isolation can effectively minimise vibrations of flexible railway vehicle bodies hence enable improved ride quality levels. Work in $[1,2]$ highlighted the importance for active suspensions and semi-active suspensions in terms of the flexible modes and their effectiveness in reducing the structural vibrations of a vehicle body. The typical active suspension system comprises front and rear suspension linear actuators. Considerable large size and power consumption are their drawbacks. Furthermore, the dynamics of the real actuators (e.g. electro-hydraulic actuators) and their strong coupling with the motion of the suspension system of the vehicle confines its performance within only a few $\mathrm{Hz}$ and there is a degradation of ride quality at higher frequencies $[3,4]$.Therefore vibration control of both the rigid modes and higher-frequency structural modes of the vehicle body with electro-hydraulic actuators is not trivial. Besides, the suspension points are near the nodes of the first bending mode, which suggest a major limitation in suppressing the flexible modes. Foo and Goodall [5] placed an electro-magnetic actuator between the centre of the vehicle and an auxiliary mass in addition to the two hydraulic actuators across the secondary suspensions for reducing the effects of the first bending mode of the vehicle body.

Another solution for vibration reduction of the flexible modes is via structural damping. The approach addresses directly the flexible structure control by integrating micro-actuator elements, e.g piezoelectric actuators, onto the flexible structure, avoiding substantial impact of added weight on the body structure. Both passive and active damping strategies for structural vibration control of railway vehicles have already been proposed. Passive structural damping was designed via shunted piezoelectric elements using electric circuits to dissipate the vibration energy of vehicle body [6, 7]. Kamada et al. [8] used piezoelectric stack transducers in shunt damping for vibration suppression of a railway vehicle. Active structural damping with piezoelectric actuators was applied by Kamada et al. [9], and independent $\mathcal{H}_{\infty}$ controllers were designed to suppress the first three flexible modes. Stack actuators mounted in consoles to introduce force/moment pairs to the structure have been proved effective for active damping of the large structure of railway vehicles $[10,11,12,13]$. Schirrer et al. [13] studied the LQG and frequency-weighted $\mathcal{H}_{2}$ control methods for vibration control of lightweight rail car body structure with piezo stack actuators, whose performances are compared with different sensor concepts. Schandl et al. [12] designed state feedback controllers by pole placement with state estimator using piezoelectric stack actuators together with collocated sensor patches for active damping of the vehicle structure. In the work by Kozek et al. [11], robust $\mathcal{H}_{\infty}$ control was applied with $\mu$ - synthesis procedure using collocated piezoelectric stack actuators and strain sensors. Benatzky et al. [10] showed that a better performance was achieved using $\mu$-synthesis procedure compared to poleplacement combined with Kalman-filter techniques for structural vibration control of a scaled metro vehicle. However, all the aforementioned structural damping strategies mentioned above did not take the suspension system into account.

To achieve effective vibration control, it is important to consider suppression of rigid mode and flexible mode vibrations simultaneously for coupling exists between 
rigid modes and flexible modes. Active structural damping, when used in addition to (active) suspension system, is expected to further improve ride quality and this facilitates retrofitting of existing railway vehicles potential [11]. Kamada et.al [14] illustrated the effectiveness of combined piezoelectric actuators and linear actuators in suppressing both the rigid and flexible modes with decentralised $\mathcal{H}_{\infty}$ controllers. However the robustness issues were not addressed in this paper, this aspect being of great significance as the system is subject to variations in parameters, actuator and sensor uncertainties and uncertainties caused by neglected high-frequency modes.

The proposed work* aims to investigate the performance and robustness of a decentralised control strategy combining active structural damping, with piezoelectric actuators and sensors, and active suspension control to suppress both the vertical rigid mode (i.e. bounce and pitch) vibrations and the vertical bending mode vibrations of the vehicle body against the track disturbances in the presence of model uncertainty. Regarding the limitation of real actuators (both electro-hydraulic actuators and piezoelectric actuators), by making sure that the two sub-controllers work in their intended frequency range, piezoelectric actuators use limited input to introduce additional damping to the structure of the vehicle body while the electro-hydraulic actuator for active suspension control focuses on the suppression of the lower frequency vibration of the rigid-body motion. The results show that the proposed scheme improves both the ride quality and the robustness of the overall system.

The remainder of the paper is organised as follows: first system modelling based on the vertical side-view of a flexible-bodied railway vehicle with piezoelectric actuators and sensors is given, and the model uncertainties are described. Then the track profile and assessment methods are introduced. Placement of actuators and sensors is addressed using $\mathcal{H}_{2}$ norms as the criteria. Next, the controller design of the decentralised controller combing active structural damping and active suspension control is presented. A brief background on $\mu$ analysis is given. In the result and discussion section, the performance and robustness of the resulting controller are presented in comparison with the controller designed with more conventional methods [15]. The effectiveness of the proposed controller in suppressing both the rigid and first bending mode of the railway vehicle in the presence of the given model uncertainties is confirmed.

\section{System description}

Here, details of the side-view model utilised for this work are presented. Firstly the rigid vehicle body and the analytical model of the flexible vehicle body are given separately. Then these are integrated to form the overall system model. Note the overall model, with the second vertical bending mode reduced, is used for controller design later in section 6. For closed-loop performance evaluation, the overall model including the first two bending modes, extended with the dynamics of the electro-hydraulic actuators with force tracking control, will be used. Further performance and stability robustness

*This paper is a substantially extended version of earlier work presented by authors in [15]. In particular, the authors extend their previous work by in-depth investigation of controller robustness, both in the design stage and expectations as well as in stability robustness analysis using $\mu$-methods to parametric uncertainty, input uncertainty (i.e. actuator uncertainty) and additive uncertainty accounting for the neglected high-frequency dynamics 


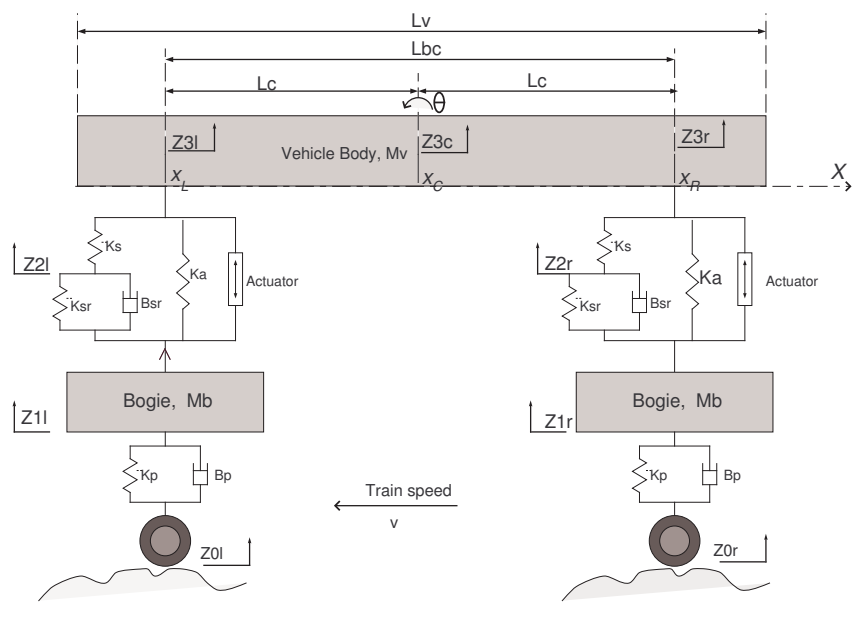

Figure 1: Sideview model of a typical high speed passenger railway vehicles

analysis incorporate model uncertainties (described in section 3).

\subsection{Side-view model of a flexible bodied railway vehicle}

The dynamics of a real railway vehicle can be very complex with non-linearities and substantial coupling in certain modes. However, it is well accepted that vertical modes are the biggest contributor to reduced ride quality: this is partly because the irregularities at relevant frequencies are substantially larger in the vertical direction, partly because the primary vertical suspension is stiffer than the lateral primary suspension, which includes the wheelset kinematic response. Therefore, the mathematical model is based on the side-view model of a railway vehicle with an air spring suspension [5], as shown in Figure 1, to suppress the vertical accelerations of the vehicle body hence improving the ride quality. In particular the side-view model in the vertical plane includes the rigid modes (i.e. bounce and pitch) and the vertical bending modes of the vehicle body as required. Since structural modes are mainly excited in the vertical suspension, there would be minimal coupling from an active vertical suspension to lateral or roll [16], and also relatively weak coupling between the vertical and lateral motions of a vehicle [17]. Low-frequency vertical vibrations may be excited by the coupling between lateral and rolling motions due to vehicle hunting and the anti-rolling bar mechanism [17], however, this is not within the scope of the analysis of this study.

The model shown in Figure 1 is a simplified model with two wheel sets included instead of four as it is sufficient to address the time delay of input excitations from front to rear bogies. The model is concerned with the stochastic vertical track inputs only, hence creep forces and conicities are not taken into account. The vehicle body is assumed as a simple elastically supported free-free beam and all the springs and dampers in the suspension are assumed to be linear. The vehicle parameters are listed in Appendix 2. The nominal value of the velocity of the vehicle, $v$, is taken to be $55 \mathrm{~m} / \mathrm{s}$. 


\subsection{Side-view model with a rigid vehicle body}

The side-view model with a rigid vehicle body includes six degrees of freedom, the bounce and pitch modes of the vehicle body, the bounce modes of the two bogie masses, and two modes associated with the internal dynamics of the air spring. The vehicle body is subject to the forces generated by the passive secondary suspension components, the springs $K_{s}, K_{a}$ and $K_{s r}$ and the damper $B_{s r}$, and the control forces from the actuators across the secondary suspensions on the left and the right, $F_{L}$ and $F_{R}$. The bogie motions are effected by both and primary suspension components, the spring $K_{p}$ and the damper $B_{p}$, and the passive secondary suspension components as well as the reaction of suspension control forces. The primary suspension is subject to the velocity disturbances from the track, $\dot{Z}_{0 L}$ and $\dot{Z}_{0 R}$. $Z_{3 C}, Z_{3 L}$, and $Z_{3 R}$ are the vertical displacements of the vehicle body measured at the vehicle centre $x_{C}$, left suspension point $x_{L}$ and right suspension point $x_{R}$ respectively, $\theta$ is the pitch angle of the vehicle body, $Z_{1 L}$ and $Z_{1 R}$ are the vertical displacements associated with the internal dynamics of the air spring in the secondary suspension, where we assume that there are two small masses, $m_{L}$ and $m_{R}$ for the convenience of modelling, and $Z_{2 L}$ and $Z_{2 R}$ are the bounce displacements of the left bogie and the right bogie respectively. The pitch angle is assumed small so that the following relationships hold

$$
\begin{aligned}
& Z_{3 L}=Z_{3 C}+L_{c} \theta \\
& Z_{3 R}=Z_{3 C}-L_{c} \theta
\end{aligned}
$$

Lagrangian equations are used to establish the equations of motion.

$$
\begin{aligned}
\frac{d}{d t}\left(\frac{\partial T}{\partial \dot{Z}_{3 C}}\right)-\frac{\partial T}{\partial Z_{3 C}}+\frac{\partial D}{\partial \dot{Z}_{3 C}}+\frac{\partial V}{\partial Z_{3 C}} & =F_{R}+F_{L} \\
\frac{d}{d t}\left(\frac{\partial T}{\partial \dot{\Theta}}\right)-\frac{\partial T}{\partial \Theta}+\frac{\partial D}{\partial \dot{\Theta}}+\frac{\partial V}{\partial \Theta} & =F_{R} L_{c}-F_{L} L_{c} \\
\frac{d}{d t}\left(\frac{\partial T}{\partial \dot{Z}_{2 L}}\right)-\frac{\partial T}{\partial Z_{2 L}}+\frac{\partial D}{\partial \dot{Z}_{2 L}}+\frac{\partial V}{\partial Z_{2 L}} & =0 \\
\frac{d}{d t}\left(\frac{\partial T}{\partial \dot{Z}_{2 R}}\right)-\frac{\partial T}{\partial Z_{2 R}}+\frac{\partial D}{\partial \dot{Z}_{2 R}}+\frac{\partial V}{\partial Z_{2 R}} & =0 \\
\frac{d}{d t}\left(\frac{\partial T}{\partial \dot{Z}_{1 L}}\right)-\frac{\partial T}{\partial Z_{1 L}}+\frac{\partial D}{\partial \dot{Z}_{1 L}}+\frac{\partial V}{\partial Z_{1 L}} & =-F_{L} \\
\frac{d}{d t}\left(\frac{\partial T}{\partial \dot{Z}_{1 R}}\right)-\frac{\partial T}{\partial Z_{1 R}}+\frac{\partial D}{\partial \dot{Z}_{1 R}}+\frac{\partial V}{\partial Z_{1 R}} & =-F_{R}
\end{aligned}
$$

in which $Z_{3 C}, \Theta, Z_{2 L}, Z_{2 R}, Z_{1 L}$ and $Z_{1 R}$ are the generalised coordinates, $T, D$ and $V$ are the total kinetic energy, dissipation function and the total potential energy of the system respectively

$$
\begin{aligned}
T= & \frac{1}{2} M v \dot{Z}_{3 C}^{2}+\frac{1}{2} I_{v c} \dot{\Theta}^{2}+\frac{1}{2} m_{L} \dot{Z}_{2 L}^{2}+\frac{1}{2} m_{R} \dot{Z}_{2 R}^{2} \\
& +\frac{1}{2} M_{b} \dot{Z}_{1 L}^{2}+\frac{1}{2} M_{b} \dot{Z}_{1 R}^{2} \\
D= & \frac{1}{2} B_{s r}\left(\dot{Z}_{2 L}-\dot{Z}_{1 L}\right)^{2}+\frac{1}{2} B_{s r}\left(\dot{Z}_{2 R}-\dot{Z}_{1 R}\right)^{2}+\frac{1}{2} B_{p}\left(\dot{Z}_{1 L}-\dot{Z}_{0 L}\right)^{2}
\end{aligned}
$$




$$
\begin{aligned}
& +\frac{1}{2} B_{p}\left(\dot{Z}_{1 R}-\dot{Z}_{0 R}\right)^{2} \\
V= & \frac{1}{2} K_{a}\left(Z_{3 L}-Z_{1 L}\right)^{2}+\frac{1}{2} K_{a}\left(Z_{3 R}-Z_{1 R}\right)^{2}+\frac{1}{2} K_{s}\left(Z_{3 L}-Z_{2 L}\right)^{2} \\
& +\frac{1}{2} K_{s}\left(Z_{3 R}-Z_{2 R}\right)^{2}+\frac{1}{2} K_{s r}\left(Z_{2 L}-Z_{1 L}\right)^{2}+\frac{1}{2} K_{s r}\left(Z_{2 R}-Z_{1 R}\right)^{2} \\
& +\frac{1}{2} K_{p}\left(Z_{1 L}-Z_{0 L}\right)^{2}+\frac{1}{2} K_{p}\left(Z_{1 R}-Z_{0 R}\right)^{2}
\end{aligned}
$$

The state-space representation of the model by equations (3)-(8) is given as follows

$$
\dot{\boldsymbol{x}}_{\boldsymbol{r}}=\boldsymbol{A}_{r} \boldsymbol{x}_{\boldsymbol{r}}+\boldsymbol{B}_{r} \boldsymbol{u}_{2}+\boldsymbol{B}_{\boldsymbol{d}} \boldsymbol{d}_{\boldsymbol{t}}
$$

where $\boldsymbol{A}_{\boldsymbol{r}}, \boldsymbol{B}_{\boldsymbol{r}}$, and $\boldsymbol{B}_{\boldsymbol{d}}$ are system matrix, suspension control input matrix , and disturbance input matrix respectively. $\boldsymbol{u}_{2}=\left[\begin{array}{ll}F_{L} & F_{R}\end{array}\right]$ is the suspension control input vector. $\boldsymbol{d}_{\boldsymbol{t}}=\left[\begin{array}{ll}\dot{Z}_{0 L} & \dot{Z}_{0 R}\end{array}\right]$ is the track velocity (disturbance) input vector. The system state vector $\boldsymbol{x}_{\boldsymbol{r}}$ is set as follows

$$
\boldsymbol{x}_{\boldsymbol{r}}=\left[\begin{array}{llllllllll}
\dot{Z}_{3 C} & \dot{\theta} & \dot{Z}_{1 L} & \dot{Z}_{1 R} & R_{1} & R_{2} & R_{3} & R_{4} & R_{5} & R_{6}
\end{array}\right]
$$

in which

$$
\begin{array}{lll}
R_{1}=Z_{3 L}-Z_{1 L} & R_{2}=Z_{3 R}-Z_{1 R} & R_{3}=Z_{2 L}-Z_{1 L} \\
R_{4}=Z_{2 R}-Z_{1 R} & R_{5}=Z_{1 L}-Z_{0 L} & R_{6}=Z_{1 R}-Z_{0 R}
\end{array}
$$

which are the relative displacements. Note that the variables $\dot{Z}_{2 R}$ and $\dot{Z}_{2 L}$ can be expressed in terms of the state variables using equations (5)-(6) when the small masses $m_{L}$ and $m_{R}$ are taken as zero.

\subsection{Analytical model of the flexible vehicle body with piezo- electric actuators and sensors}

The flexible vehicle body is assumed as a free-free Euler-Bernoulli beam of length $l$, cross-section area $A_{b}$, mass $M_{v}$, material density $\rho_{b}$, and flexural rigidity $E I$, which depends on the Young's modulus of elasticity $E$ and the second moment of area $I$. The flexural vibration of the beam is induced by track disturbances transmitted through the suspensions. Stack piezoelectric actuators mounted in consoles as shown in Figure 2 are bonded onto the vehicle body for suppressing the flexible modes. We choose this type of actuator because it is capable of introducing the necessary large bending moments concerning the size, mass and stiffness of the structure [18].

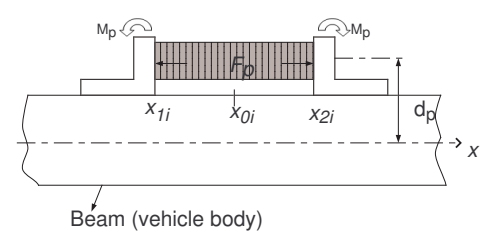

Figure 2: Piezoelectric actuator

The actuator expands when a voltage $V_{a}$ is applied to it and actuation force $F_{p}$ is generated when this expansion is constrained by mounting the actuator in a mechanical 
structure. It assumes that the equivalent actuation force is proportional to the applied voltage

$$
F_{p}=A_{p} e_{33} \frac{V_{a}}{h_{S t}}
$$

where $e$ is the piezoelectric coupling matrix, $h_{S t}$ is the thickness of one disk, and $A_{p}$ is the area of cross-section of the actuator.

The governing partial differential equation (PDE) that describes the dynamics of the structure of the beam, assuming the beam is one-dimension only and has zero damping, is given as follows [19]

$$
E I \frac{\partial^{4} z(x, t)}{\partial x^{4}}+\rho_{b} A_{b} \frac{\partial^{2} z(x, t)}{\partial t^{2}}=\sum_{i=1}^{n_{p}} \frac{\partial^{2} M_{p x}^{i}(x, t)}{\partial x^{2}}+F_{x_{L}}(t) \delta\left(x-l_{1}\right)+F_{x_{R}}(t) \delta\left(x-l_{2}\right)
$$

where $z(x, t)$ is the transverse deflection at point $x$ of the beam and time $t, F_{x_{L}}$ and $F_{x_{R}}$ are the forces acting at the left and right suspension points. $i=1,2 \ldots, n_{p}$ denotes the number of piezoelectric actuators, $M_{p x}^{i}$ is the moment acting on the beam by the piezoelectric actuators and is given by

$$
M_{p x}^{i}(x, t)=k_{a} V_{a i}\left[H\left(x-x_{1 i}\right)-H\left(x-x_{2 i}\right)\right]
$$

where $V_{a i}$ is the voltage applied to the $i$ th actuator. $H($.$) is the step function. x_{1 i}$ and $x_{2 i}$ denote the location of the two ends of the $i$ th piezoelectric actuator along the $X$ axis, and $k_{a}$ is a constant based on the properties of the beam and the piezoelectric actuator

$$
k_{a}=\frac{A_{p} e_{33} d_{p}}{h_{s t}}
$$

where $d_{p}$ is is the vertical distance from the neutral axis of the vehicle body to the vertical centre of the piezoelectric actuator.

By modal analysis technique, the solution of equation (16) is can be written as

$$
z(x, t)=Z_{3 C}(t)+\theta(t)\left(x-L_{b} / 2\right)+\sum_{r=1}^{\infty} q_{r}(t) \phi_{r}(x)
$$

where $Z_{3 C}(t)$ and $\theta(t)$ are the bounce mode and pitch mode respectively, $q_{r}(t)$ is the $r$ th flexible mode in the generalised coordinate and $\phi_{r}(x)$ is the corresponding mode shape, which can be determined from the eigenvalue problem of a free-free beam [15]. Figure 3 shows the mode shapes of the first two vertical bending modes.

For practical reasons, it is not possible to include in the model an infinite number of modes. Only the first vertical bending mode will be included in the model for controller design for it has shown the most significant influence on the ride quality of the railway vehicle $[5,3]$, while the first two vertical bending modes are included in the model for analysis and simulation of both the passive system and the closedloop system with the designed controllers. Using the orthogonality properties of the mode shapes and Dirac's delta function property, the uncoupled ordinary differential equations representing the motions of the first two vertical bending modes of the beam can be obtained by integrating equation (16) over the beam length [15].

$$
\ddot{q}_{r}(t)+2 \zeta_{r} \omega_{r} \dot{q}_{r}(t)+\omega_{r}^{2} q_{r}(t)=\frac{k_{a}}{M_{v}} \sum_{i=1}^{n_{p}} \Psi_{a r i} V_{a i}(t)+\frac{F_{x_{L}} \phi_{r}\left(x_{L}\right)}{M_{v}}+\frac{F_{x_{R}} \phi_{r}\left(x_{R}\right)}{M_{v}} \quad r=1,2
$$


where structural damping is added and $\zeta_{r}$ is the damping ratio of the $r^{\text {th }}$ flexible mode, and $\Psi_{\text {ari }}$ is for the $r^{\text {th }}$ mode and the $i^{\text {th }}$ piezoelectric actuator and is given by

$$
\Psi_{\text {ari }}=\frac{d \phi_{r}}{d x}\left(x_{2 i}\right)-\frac{d \phi_{r}}{d x}\left(x_{1 i}\right)
$$

According to the force terms at the right-hand side of equation (20), the absolute value of a mode shape at certain point is an evaluation of effectiveness of the control force acting at that point, and suspension control is not considered efficient in suppressing the first bending model because the two suspension points are near the nodes of this mode, i.e. the positions where the corresponding mode shapes are zero, as is shown by figure 3 .

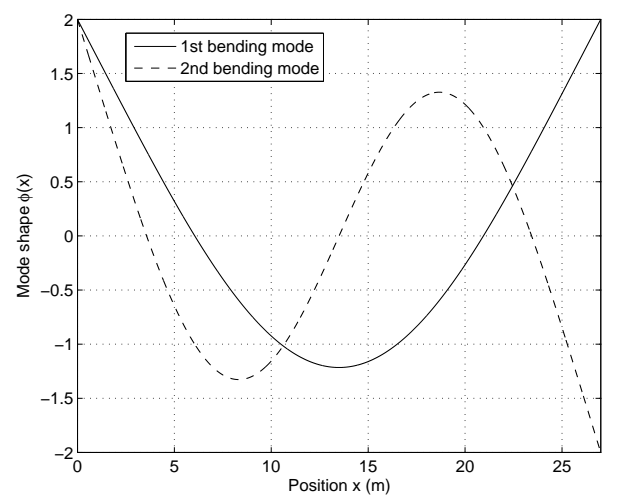

Figure 3: Mode shapes of the free-free beam of a railway vehicle

Piezoelectric laminar sensors are bonded to the structure for measuring the flexible modes. They produce voltage signals proportional to the average curvature. They are advantageous when only the vibrations of the flexible modes have to be measured and the signal part of the rigid body modes has to be eliminated [10]. The induced voltage $v_{s j}$ in the $j^{t h}$ piezoelectric sensor caused by the first two bending modes is given as [19]

$$
v_{s j}(t)=k_{s} \sum_{r=1}^{2} \Psi_{s r j} q_{r}(t)
$$

where $k_{s}$ is a constant related to the properties of the sensor and the beam, $\Psi_{s r j}$ is for the $r^{\text {th }}$ mode and the $j^{\text {th }}$ piezoelectric sensor,

$$
\Psi_{s r j}=\frac{d \phi_{r}}{d x}\left(x_{2 j}\right)-\frac{d \phi_{r}}{d x}\left(x_{1 j}\right) \quad r=1,2
$$

in which $x_{1 j}$ and $x_{2 j}$ denote the position of the two ends of the $j^{\text {th }}$ sensor.

The state-space representation of the flexible motions of equation (20) is given as follows

$$
\begin{aligned}
\dot{x_{f}} & =\boldsymbol{A}_{f} \boldsymbol{x}_{f}+\boldsymbol{B}_{f 1} \boldsymbol{u}_{1}+\boldsymbol{B}_{f 2} d \\
\boldsymbol{y}_{1} & =\boldsymbol{C}_{\boldsymbol{v}} \boldsymbol{x}_{f}
\end{aligned}
$$

where the state vector $\boldsymbol{x}_{\boldsymbol{f}}(t)$ is chosen as

$$
\boldsymbol{x}_{\boldsymbol{f}}(t)=\left[\begin{array}{llll}
\dot{q}_{1}(t) & q_{1}(t) & \dot{q}_{2}(t) & q_{2}(t)
\end{array}\right]
$$


$\boldsymbol{u}_{\mathbf{1}}$ is the $\left(n_{p} \times 1\right)$ vector of voltage inputs to the piezoelectric actuators, $\boldsymbol{d}=\left[F_{x_{L}} F_{x_{R}}\right]$ is the vector of forces acting at the suspension points

$$
\begin{aligned}
& F_{x_{L}}=K_{s}\left(Z_{3 L}-Z_{2 L}\right)+F_{L} \\
& F_{x_{R}}=K_{s}\left(Z_{3 R}-Z_{2 R}\right)+F_{R}
\end{aligned}
$$

$\boldsymbol{y}_{\mathbf{1}}$ is the $\left(m_{p} \times 1\right)$ vector of measured variables by piezoelectric sensors, and the corresponding input matrices and output matrices are as following

$$
\begin{aligned}
\boldsymbol{B}_{\boldsymbol{f} \mathbf{1}}= & \frac{k_{a}}{M_{v}}\left[\begin{array}{cccc}
\Psi_{a 11} & \Psi_{a 12} & \cdots & \Psi_{a 1 n_{p}} \\
0 & 0 & \cdots & 0 \\
\Psi_{a 21} & \Psi_{a 22} & \cdots & \Psi_{a 2 n_{p}} \\
0 & 0 & \cdots & 0
\end{array}\right] \\
\boldsymbol{B}_{\boldsymbol{f} \mathbf{2}}= & {\left[\begin{array}{cc}
\frac{\phi_{1}\left(x_{L}\right)}{M_{v}} & \frac{\phi_{1}\left(x_{R}\right)}{M_{v}} \\
0 & 0 \\
\frac{\phi_{2}\left(x_{L}\right)}{M_{v}} & \frac{\phi_{2}\left(x_{R}\right)}{M_{v}} \\
0 & 0
\end{array}\right] } \\
\boldsymbol{C}_{\boldsymbol{v}}= & \frac{k_{s}}{C_{p}}\left[\begin{array}{cccc}
0 & \Psi_{s 11} & 0 & \Psi_{s 21} \\
0 & \Psi_{s 12} & 0 & \Psi_{s 22} \\
0 & \vdots & 0 & \vdots \\
0 & \Psi_{s 1 m_{p}} & 0 & \Psi_{s 2 m_{p}}
\end{array}\right]
\end{aligned}
$$

\subsection{The model of the overall system}

The overall model is formed by integrating the side-view model with rigid vehicle body (12) and the model of the flexible vehicle body (24) by introducing the couplings between the states of the two models. The couplings between the rigid motion and the flexible motion of a flexible structure are introduced by control (either active or passive) and takes places in two ways, actuation and sensing [20]. As for the passive system represented by the side-view model of the railway vehicle, the couplings between the rigid motion and the flexible motion are introduced by the passive elements directly connected to the vehicle body, the springs $K_{s}$ and $K_{a}$, which are viewed as collocated sensors and actuators. The absolute vertical displacements sensed by springs $K_{s}$ and $K_{a}$ at positions $x_{L}$ and $x_{R}$ include not only the bounce and pitch of the vehicle body but also the first two vertical bending modes of the vehicle body

$$
\begin{aligned}
& Z_{3 L}=Z_{3 C}+\theta(t) L_{c}+q_{1}(t) \phi_{1}\left(x_{L}\right)+q_{2}(t) \phi_{a}\left(x_{L}\right) \\
& Z_{3 R}=Z_{3 C}-\theta(t) L_{c}+q_{2}(t) \phi_{1}\left(x_{R}\right)+q_{2}(t) \phi_{a}\left(x_{R}\right)
\end{aligned}
$$

which in turn produce forces that affect both the rigid and flexible motions of the vehicle body.

Therefore, the state-space representation of the overall model could be obtained from equations (12) and (24) with the vertical displacements sensed by springs $K_{s}$ and $K_{a}$ replaced by equations (31) and (32)

$$
\dot{\boldsymbol{x}}=\left[\begin{array}{cc}
\boldsymbol{A}_{r}(12 \times 12) & \boldsymbol{A}_{\boldsymbol{f} \boldsymbol{r}}(12 \times 4) \\
\boldsymbol{A}_{\boldsymbol{r} \boldsymbol{f}}(4 \times 12) & \boldsymbol{A}_{\boldsymbol{f}}(4 \times 4)
\end{array}\right] \boldsymbol{x}+\left[\begin{array}{c}
\boldsymbol{B}_{\boldsymbol{r}} \\
\boldsymbol{B}_{\boldsymbol{f} \mathbf{1}}
\end{array}\right] \boldsymbol{u}_{\boldsymbol{1}}
$$




$$
+\left[\begin{array}{c}
0 \\
B_{f 2}
\end{array}\right] u_{2}+\left[\begin{array}{c}
B_{d} \\
0
\end{array}\right] d_{t}
$$

in which $\boldsymbol{x}$ is the state vector of the overall model

$$
\boldsymbol{x}=\left[\begin{array}{ll}
\boldsymbol{x}_{\boldsymbol{r}} & \boldsymbol{x}_{f}
\end{array}\right]
$$

and $\boldsymbol{A}_{\boldsymbol{f} \boldsymbol{r}}$ and $\boldsymbol{A}_{\boldsymbol{r} \boldsymbol{f}}$ are the coupling matrices between the rigid states $\boldsymbol{x}_{\boldsymbol{r}}$ and the flexible states $\boldsymbol{x}_{\boldsymbol{f}}$.

Based on the parameters given in Appendix 2, model analysis shows that the bounce and pitch modes of the vehicle body are at frequencies of 4.2 and $5.3 \mathrm{rad} / \mathrm{s}$ with damping ratios of 0.16 and 0.2 , respectively and the first bending mode of the vehicle body is at $53.1 \mathrm{rad} / \mathrm{s}$ and the second bending mode is around $145.8 \mathrm{rad} / \mathrm{s}$. Since the highest sensitivity of a human is within the frequency range of $25-63 \mathrm{rad} / \mathrm{s}$, it is important to suppress the first bending mode in order to improve ride comfort.

\section{Uncertainty Analysis}

Due to the assumptions and simplifications made during modelling in section 2 (i.e. free-free Euler-Bernoulli assumption of the vehicle body, perfect bonding of the actuators, linear elasticity, linear actuator and sensor dynamics, ignorance of irrelevant and high-frequency dynamics), a degree of uncertainty is contained in the model derived in section 2. Moreover, the system is subject to load changes which in turn cause variations in vehicle mass, pitch inertia, and damping and frequency of the flexible modes. This poses a great challenge for control design. In this study, the controller is designed with the simplified model so that the control efforts concentrate on the specific frequency range of the rigid modes and the the first bending mode of the vehicle body, thus having minimum influence over the frequencies where the model is uncertain. Then the performance and stability robustness of the closed-loop system will be evaluated against the model uncertainties. The parametric uncertainties, uncertain actuator dynamics and neglected flexible modes are perceived as the major sources of uncertainty of the system and will be modelled in this section.

\subsection{Parametric uncertainties}

Load variation is perceived as the main cause for parametric uncertainty. Passenger loading behaves as additional damping elements upon the vehicle body flexural vibrations with little change on the natural frequencies [21]. Three realistic cases, as shown by Table 1, are investigated, which are named by increased passenger load, decreased stiffness of vehicle body and stiffened vehicle body, with each involving a combinational variations of parameters [11]. The parametric uncertainty here is represented in the form of multiplicative perturbation, which is an easy way for modelling the relative variations of parameters.

$$
\alpha_{i p}=\bar{\alpha}_{i}\left(1+W_{i} \delta_{i}\right)
$$

where $\delta_{i}$ is any real scalar satisfying $\left|\delta_{i}\right|<1$, and the factor $W_{i}$ defines the magnitude of the relative uncertainties. 
Table 1: Parametric uncertainties

\begin{tabular}{|l|c|c|c|c|}
\hline Testing Cases & $\Delta M_{v}$ & $\Delta I_{v c}$ & $\Delta \omega_{s}$ & $\Delta \zeta_{s}$ \\
\hline Increased passenger load & $+50 \%$ & $+20 \%$ & - & $+800 \%$ \\
Decreased stiffness & - & - & $-20 \%$ & $-25 \%$ \\
Increased stiffness & - & - & $+20 \%$ & $+10 \%$ \\
\hline
\end{tabular}

\subsection{Input multiplicative uncertainties}

For each control input $\boldsymbol{u}_{i}$, a separate physical system (including actuator, amplifier, valve,etc) is always associated, which leads to the ever present uncertainties in each individual input channel. Diagonal input multiplicative uncertainty could be used to model this type of uncertainty, by which relative uncertainty is defined for each input channel [22]

$$
\boldsymbol{G}_{p}(s)=\boldsymbol{G}(s)\left(\boldsymbol{I}+\boldsymbol{W}_{m}(s) \boldsymbol{\Delta}_{m}(s)\right)
$$

where $\boldsymbol{G}_{p}$ is the transfer function of the perturbed model, $\boldsymbol{\Delta}_{m}(s)=\operatorname{diag}\left\{\delta_{i}(s)\right\}$ is the diagonal normalised uncertainty satisfying $\left|\delta_{i}\right| \leq 1$, and $\boldsymbol{W}_{m}(s)=\operatorname{diag}\left\{W_{m i}\right\}$ is the diagonal weighting function matrix and $W_{m i}$ defines the size of relative uncertainty over frequency for the $i^{\text {th }}$ input channel.

For the input channel of each electro-hydraulic actuator, the weighting function $W_{m 1}$ is chosen in the form as follows [22]

$$
W_{m 1}=\frac{0.05 s+0.2}{(0.05 / 4) s+1}
$$

whose gain is 0.2 at steady state and reaches $100 \%$ at $20 \mathrm{rad} / \mathrm{s}$, while reaching $400 \%$ at high frequencies. This is according to the results in [3] that the hydraulic actuator is able to track the low-frequency demands only until about $20 \mathrm{rad} / \mathrm{s}$, and unsatisfactory performance begins to emerge at higher frequencies due to the limited bandwidth of the real actuators and their strong coupling with the motion of the suspension system of the vehicle .

The relative uncertainty associated with the input channel of each piezoelectric actuator is considered at most $2 \%$, constant over the frequency range, to account for the nonlinear hysteresis behaviour of the voltage-force relation of the piezoelectric actuators, as the frequency range is uncritical for the variation of the actuator gain [23], hence the weighting function $W_{m 2}$ is chosen to be

$$
W_{m 2}=0.02
$$

\subsection{Additive uncertainty}

The free-free beam approximation of the flexural vibration of the vehicle body takes into account only the first two vertical bending modes. The effects of the diagonal distortion mode and torsional mode, which are in the frequency range of human sensitivity, and localised high-frequency deformation caused by structure actuation $[13,11]$, are neglected. Moreover, measurement tests of the actual railway vehicles show the complicated the roof and floor in-phase and anti-phase bending modes [24] and the 
simulation of a virtual vehicle system shows additional mode shapes due to the elastically mounted equipments [25]. Hence, additive uncertainty is used to represent the neglected complex flexural dynamics and high-frequency modes of the railway vehicle body, for the additive uncertainty in frequency domain is regarded an intuitive representation for the complex neglected and unmodelled uncertainties, which are often less precise and difficult to quantify [22].

$$
\boldsymbol{G}_{p}=\boldsymbol{G}(s)+\boldsymbol{W}_{a}(s) \boldsymbol{\Delta}_{a}(s),\left\|\boldsymbol{\Delta}_{a}(s)\right\|_{\infty} \leq 1
$$

where $\boldsymbol{G}_{p}$ is the transfer function of the perturbed model, $\boldsymbol{\Delta}_{a}(s)$ is the normalised additive uncertainty, $\boldsymbol{W}_{a}(s)$ is the weighting function that determines the size of the additive uncertainty over frequency and is chosen based on the principle that the uncertainty is small where the model for controller design is relatively accurate and is large where no information about the system is contained in the model and take the form as follows $[10,26]$

$$
\boldsymbol{W}_{a}(s)=\boldsymbol{L}_{a} \frac{\left(s / \omega_{c 1}+1\right)^{2}}{\left(s / \omega_{c 2}+1\right)^{2}}
$$

which is a a second order filter to ensure fast roll-off at $40 \mathrm{~dB} /$ decade. $\boldsymbol{L}_{a}$ is a constant matrix which reflects the relative gains for each entry of the uncertainty matrix, and is chosen according to the steady-state gains of the passive system. The corner frequencies $\omega_{c 1}=38 \mathrm{rad} / \mathrm{s}$ and $\omega_{c 1}=94 \mathrm{rad} / \mathrm{s}$ are chosen so that the additive uncertainty is small at frequencies smaller than $\omega_{c 1}$ and increases to the maximum at $\omega_{c 2}$, considering the unmodelled diagonal distortional mode and torsional mode in the frequency range $\omega \in\left[\omega_{c 1}, \omega_{c 2}\right]$ and higher frequency modes [11].

To aggregate all model uncertainties, we can define fictitious inputs and outputs connected to each normalised uncertainty, pull all sources of uncertainty out of the model and collect them in a single diagonal matrix uncertainty. Hence we are able to manipulate the system model into a general structure for robustness analysis, which will be explained more in detail in Section 7.

\section{Placement of piezoelectric actuators and sensors}

The placement of actuators and sensors is very important in achieving efficient control of flexible structures. As normally only a reduced model is needed for control design, one prefers to have high level of controllability and observability of the modes to be controlled, and at the same time low level of controllability and observability for the residual modes, for high-frequency noise rejection and reducing spillover effect. A number of criterion exist that reflect the controllability and observability measures $[27,28,29]$.

In this paper we follow the methodology given in [28]. The $\mathcal{H}_{2}$ structural norm is used as an evaluation metric to provide an analytical tool integrated to the control method adopted, the frequency-weighted $\mathcal{H}_{2}$ control, for structural damping. For the model in modal coordinate, the $\mathcal{H}_{2}$ norm of the $r^{\text {th }}$ mode, $i^{\text {th }}$ actuator and $j^{\text {th }}$ sensor is approximately determined from

$$
\left\|G_{r i j}\right\|_{2} \cong \frac{\left\|b_{r i}\right\|_{2}\left\|c_{r j}\right\|_{2}}{2 \sqrt{\zeta_{r} \omega_{r}}}
$$


where $b_{r i}$ is the input matrix for the $r^{t h}$ mode and the $i^{\text {th }}$ actuator,$c_{r j}$ is the output matrix for the $r^{t h}$ mode and the $j^{\text {th }}$ sensor, and $\zeta_{r}$ and and $\omega_{r}$ are the damping ratio and natural frequency of the the $r^{t h}$ mode respectively. The $\mathcal{H}_{2}$ norm of the $r^{\text {th }}$ mode with a set of $n_{p}$ actuators and $m_{p}$ sensors is the root-mean-square sum of $\mathcal{H}_{2}$ norm of the mode over all actuators.

$$
\left\|G_{r}\right\|_{2} \cong \sqrt{\sum_{i=1}^{n_{p}}\left\|G_{r i}\right\|_{2}^{2}}
$$

Figure 4 shows the normalised $\mathcal{H}_{2}$ indices as a function of actuator location with a fixed sensor. The norm for the first bending mode reaches maxima at the centre of the vehicle body $\mathrm{x}=(13.5)$ and reduces to zero as the actuator approaching the two ends of the vehicle body. The norm of the second bending mode reaches maxima at positions $\mathrm{x}=(7.84)$ and $\mathrm{x}=(19.16)$ and is zero at the centre of the vehicle body $\mathrm{x}=(13.5)$. Hence, it is preferable to place the piezoelectric actuators near the centre position and away from positions $\mathrm{x}=(7.84)$ and $\mathrm{x}=(19.16)$, where most effective control can be achieved for the first bending mode with $\mathcal{H}_{2}$ control while having minimum effect on the second bending mode.

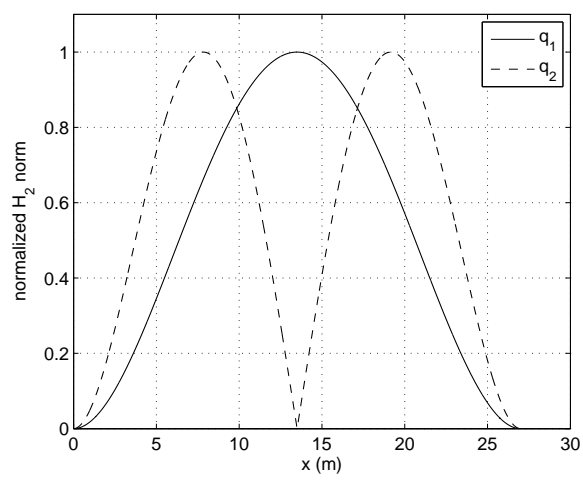

Figure 4: Normalized $\mathcal{H}_{2}$ indices as a function of actuator location with fixed sensor

The optimal placement of actuators and sensors is concerned with determining a smaller subset of actuators and sensors that are as close as possible, performancewise, to that of the larger set of actuators and sensors, so as to chose a smallest set of actuators and sensors necessary. Figure 5 shows the configuration of piezoelectric actuators and sensors in this study. Three piezoelectric stack actuators (20 in parallel) are placed around the centre position $x=(13.5)$ to attain the necessary moments, and one patch sensor are placed at position $x=(13.5)$ for measuring the motion of the first bending mode. The actuator and sensor at the centre are chosen to coincide to achieve better robustness properties. The corresponding $\mathcal{H}_{2}$ norms are $1.75 \times 10^{-6}$ for the first bending mode and $2.6 \times 10^{-13}$ for the second bending mode, hence it is expected to be effective in suppressing the first bending mode and have little influence on the second bending mode. 


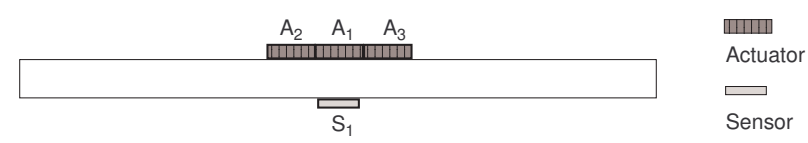

Figure 5: Configuration of piezoelectric actuators and sensors

\section{Track profile and assessment method}

The assessment of ride quality relates to vehicle running on straight track with vertical misalignments (irregularities). The wheelsets are connected directly to dampers and this necessitates track velocities as inputs to the system. For secondary suspension assessment, Gaussian white noise with a flat spectrum is a good approximation for the track velocity inputs [30]. Hence the track velocity spectrum or the derivative of the track displacement spectrum is given by

$$
\dot{S}_{T}\left(\omega_{t}\right)=2 \pi \Omega_{v} v \quad\left(m s^{-1}\right)^{2}(\mathrm{rad} / \mathrm{s})^{-1}
$$

where $\Omega_{v}$ is the roughness factor, which is taken as $2.5 \times 10^{-7}$ for a good quality mainline track.

Root mean square (RMS) with frequency weighting to account for human susceptibility is often used to quantify the ride quality. In this case unweighted RMS values of vertical accelerations of the vehicle body are calculated so as to not to obscure any undesirable effects. Covariance method is used to evaluate the RMS values [3]. And the power spectral densities (PSDs) of the signals of interest, including body accelerations $\left(\ddot{Z}_{3 L}, \ddot{Z}_{3 C}\right.$ and $\left.\ddot{Z}_{3 R}\right)$ and suspension deflections $\left(Z_{3 L}-Z_{1 L}\right.$ and $\left.Z_{3 R}-Z_{1 R}\right)$ in response to track irregularities, are evaluated by frequency domain method, the output power spectrum is given by

$$
S_{y}=\left|H_{f}(j \omega)+H_{r}(j \omega) e^{-j \omega t_{\text {delay }}}\right|^{2} \dot{S}_{T}\left(\omega_{t}\right)
$$

where $H_{f}(j \omega)$ and $H_{r}(j \omega)$ are respectively the transfer function from the front and rear velocity input to the output. $t_{\text {delay }}$ is the delay between the front and rear track input, which is taken as $0.35 \mathrm{~s}$ in this case.

\section{Controller design}

The controller design aims to improve the ride quality of the railway vehicle by reducing the vertical accelerations of the vehicle body against vertical track irregularities while maintaining the suspension deflections as well as control inputs within certain limits for practical reasons. It must be able to do so in the presence of model uncertainties.

To keep the controller simple, a decentralised control strategy is proposed as shown by Figure 6, where $\boldsymbol{G}(s)$ is model for control design, $\boldsymbol{K}_{\text {stru }}$ is the structural damping controller that utilises piezoelectric actuators and sensors targeting the flexible modes, $\boldsymbol{u}_{\mathbf{1}}$ is vector of control inputs to piezoelectric actuators, $\boldsymbol{y}_{\mathbf{1}}$ is vector of measurement outputs by the piezoelectric sensors, $\boldsymbol{K}_{\text {sus }}$ is the suspension controller, $\boldsymbol{u}_{\mathbf{2}}$ is the vector of control inputs for suspension control, $\boldsymbol{y}_{2}$ is vector of measured accelerations of the 


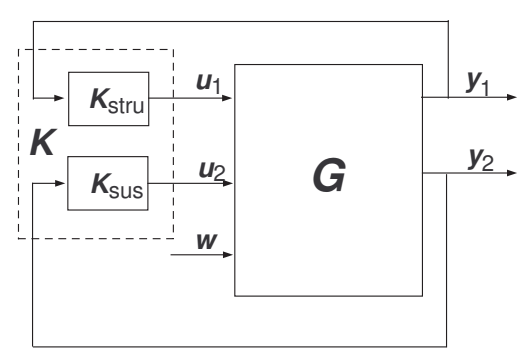

Figure 6: Decentralised control structure Figure 7: Frequency-weighted $\mathcal{H}_{2}$ control in generalised framework

vehicle body, and $\boldsymbol{w}$ is the vector of exogenous inputs including the track inputs and measurement noises.

The two sub-controllers are designed in a sequential manner. Firstly, $\boldsymbol{K}_{\text {stru }}$ is designed using the model of the flexible vehicle body including only the first vertical bending mode. Then $\boldsymbol{K}_{\text {sus }}$ is designed with the controller $\boldsymbol{K}_{\text {stru }}$ applied, using the overall model with the second bending mode reduced. Both the electro-hydraulic actuators and piezoelectric actuators are assumed to be ideal for controller design. The resulting decentralised controller is given by

$$
\boldsymbol{K}=\left[\begin{array}{cc}
\boldsymbol{K}_{\text {stru }} & 0 \\
0 & \boldsymbol{K}_{\text {sus }}
\end{array}\right]
$$

\subsection{Active structural damping}

The control design for active structural damping with piezoelectric actuators and sensors is required to reduce the vertical accelerations of the vehicle body caused by the flexural vibrations. The model of the flexible vehicle body considering only the first bending mode is used to make the control efforts focus on the first bending mode, which is the main flexural mode that causes reduced ride quality. Frequency-weighted $\mathcal{H}_{2}$ control is adopted because $\mathcal{H}_{2}$ control is considered particularly suited for vibration control problems in which the system is subjected to disturbances of broadband or of known spectral content, and the $\mathcal{H}_{2}$ norm is a more natural measurement of performance in treatment of disturbances [31]. Moreover, we are able to use the dynamic weighting functions to shape the controller actions within the specific target frequency ranges so as to have minimum influence on modes of other frequencies [13]. Hence the controller is expected to be less sensitive to model uncertainties. $\mathcal{H}_{2}$ control is a generalization of the LQG control but can consider the performance more explicitly. The performance of the proposed controller will be compared with that of an LQG controller in section 8 .

\subsubsection{Frequency-weighted $\mathcal{H}_{2}$ control design}

Figure 7 shows the frequency-weighted $\mathcal{H}_{2}$ control for active structural damping of the railway vehicle in the generalised framework. $\boldsymbol{u}_{1}$ is the vector of control inputs, $\boldsymbol{n}$ is the vector of sensor noises, $\boldsymbol{d}$ is the vector of disturbances that models the excitation 
acting at the two suspension points, $\boldsymbol{w}_{\boldsymbol{f}}$ is the vector of scaled exogenous signals, $\boldsymbol{z}_{\boldsymbol{f}}$ is the vector weighted regulated variables, which include the regulated output $\boldsymbol{y}_{p}$ and the control inputs $\boldsymbol{u}_{\mathbf{1}}, \boldsymbol{y}_{\mathbf{1}}$ is the vector of measured outputs. $\boldsymbol{G}_{\boldsymbol{f}}$ is the design model, which is the model of the flexible vehicle body including only the first vertical bending mode. The generalised plant $\boldsymbol{P}$ includes $\boldsymbol{G}_{\boldsymbol{f}}$, the interconnection, and the matrix weighting functions $\boldsymbol{W}_{\boldsymbol{d}}, \boldsymbol{W}_{\boldsymbol{n}}, \boldsymbol{W}_{\boldsymbol{u}_{\boldsymbol{1}}}$ and $\boldsymbol{W}_{\boldsymbol{y}}$, which are to be specified to meet the control objectives. Hence control problem is manipulated into the general problem formulation written as follows [22].

$$
\begin{aligned}
{\left[\begin{array}{c}
\boldsymbol{z}_{\boldsymbol{f}} \\
\boldsymbol{y}_{\mathbf{1}}
\end{array}\right] } & =P(s)\left[\begin{array}{c}
\boldsymbol{w}_{\boldsymbol{f}} \\
\boldsymbol{u}_{\mathbf{1}}
\end{array}\right]=\left[\begin{array}{l|l}
P_{11} & P_{12} \\
\hline P_{21} & P_{22}
\end{array}\right]\left[\begin{array}{l}
\boldsymbol{w}_{\boldsymbol{f}} \\
\boldsymbol{u}_{\mathbf{1}}
\end{array}\right] \\
\boldsymbol{u}_{\mathbf{1}} & =\boldsymbol{K}_{\text {stru }}(s) \boldsymbol{y}_{\mathbf{1}}
\end{aligned}
$$

The control design aims to find a stabilizing controller $\boldsymbol{K}_{\text {stru }}$ that minimizes the $\mathcal{H}_{2}$ norm of the closed-loop transfer function $\boldsymbol{T}_{\boldsymbol{w}_{\boldsymbol{f}} \boldsymbol{z}_{\boldsymbol{f}}}$, which can be readily solved with the commercial packages in Matlab. The resulting controller is the combination of an optimal linear-quadratic regulator and a Kalman-Bucy observer solved by two Riccati equations, the interested reader is referred to [22] and [32] for more details. In addition, for the design of the gains of the regulator and the estimator, there is a trade-off between performance and robustness.

The regulated output $\boldsymbol{y}_{\boldsymbol{p}}$ includes the vertical accelerations caused by the first bending mode at three positions, centre of the vehicle body and left and right suspension points on the vehicle body .

$$
\boldsymbol{y}_{p}=\left[\ddot{Z}_{f}\left(x_{C}\right) \ddot{Z}_{f}\left(x_{L}\right) \ddot{Z}_{f}\left(x_{R}\right)\right]
$$

where $Z_{f}(x)=q_{1}(x) \phi_{1}(x)$. The dynamic weighting matrix $\boldsymbol{W}_{\boldsymbol{y}}$ is specified to produce an increased gain around the frequency of the first bending mode $(52.8 \mathrm{rad} / \mathrm{s})$ so as to reduce the frequency response of the closed-loop system in this region

$$
\boldsymbol{W}_{\boldsymbol{y}}=k_{y} \frac{s^{2}+2 \zeta_{y a} \omega_{y} s+\omega_{y}^{2}}{s^{2}+2 \zeta_{y b} \omega_{y} s+\omega_{y}^{2}} \boldsymbol{I}_{\mathbf{3} \times \mathbf{3}}
$$

where $\omega_{y}=52.8 \mathrm{rad} / \mathrm{s}, \zeta_{y a}=0.7, \zeta_{y b}=0.3$. The control voltages applied to the piezoelectric actuators are also regulated for it is necessary to limit the size of control effort and constrain its frequency content within the neighbourhood of the first bending mode so as to make more efficient use of the piezoelectric actuators as well as avoid any spill-over effect. The weighing matrix $\boldsymbol{W}_{u 1}$ is specified as

$$
\boldsymbol{W}_{\boldsymbol{u} \mathbf{1}}=k_{u 1} \frac{s^{2}+2 \zeta_{u 1 a} \omega_{u 1} s+\omega_{u 1}^{2}}{s^{2}+2 \zeta_{u 1 b} \omega_{u 1} s+\omega_{u 1}^{2}} \boldsymbol{I}_{\mathbf{3} \times \mathbf{3}}
$$

where $\omega_{u 1}=52.8 \mathrm{rad} / \mathrm{s}, \zeta_{u 1 a}=0.2, \zeta_{u 1 b}=0.7$. The coefficients $k_{y}$ and $k_{u 1}$ of the weighting functions of the regulated variables are tuned to balance the overall performance and the control efforts.

The weighting function $\boldsymbol{W}_{\boldsymbol{d}}$ matrix is specified to capture the spectrum of the excitation forces in vector $\boldsymbol{d}$ acting at the two suspension points, with a second-order 
lag function with cut-off frequency $\omega_{c}=15 \mathrm{rad} / \mathrm{s}$ according to the simulation of the passive system

$$
\boldsymbol{W}_{\boldsymbol{d}}=k_{d} \frac{225}{s^{2}+30 s+225} \boldsymbol{I}_{\mathbf{2} \times \mathbf{2}}
$$

The weighting function $W_{n}$ characterises the variance of the sensor noise, hence is chosen as a constant. As the measurement noise is very small compared to the disturbance input, a high ratio of $k_{d} / W_{n}$ is used.

\subsection{Active suspension control}

The design of active suspension controller aims to reduce the vibration of the rigid modes (bounce and pitch) of the vehicle body while maintaining adequate suspension deflections with electro-hydraulic actuators, which are of high power-to-weight ratio and low cost and considered most viable to be fitted between the vehicle body and the bogie. The controller is designed with the structural damping controller $\boldsymbol{K}_{\text {stru }}$ applied, using the overall model with the second bending mode reduced, as is shown by Figure 9. Modal control with skyhook damping, with its gains tuned by structured $\mathcal{H}_{\infty}$ synthesis technique, is adopted, by which we desire to keep the controller simple while achieving more complex control goals, i.e. good performance and robustness in face of the limited bandwidth of the actuators when coupled with the railway suspension system and model uncertainties.

\subsubsection{Modal control with skyhook damping}

The advantage of skyhook damping lies in its simplicity. It is the conventional method known to give sufficient improvements in the ride quality of railway vehicles. Figure 8 shows the control scheme of modal control with skyhook damping for the railway system.

Two linear accelerometers are used to measure vehicle body accelerations at the two suspension points $x_{L}$ and $x_{R}, \ddot{Z}\left(x_{L}\right)$ and $\ddot{Z}\left(x_{R}\right)$. The measured signals are decomposed into modal components (i.e. bounce $\ddot{Z}_{b}$ and pitch $\ddot{Z}_{p}$ ). Then the bounce and pitch modes are controlled separately with skyhook damping, and finally the modal control signals are recombined and feed to the actuators. The control action of an ideal skyhook

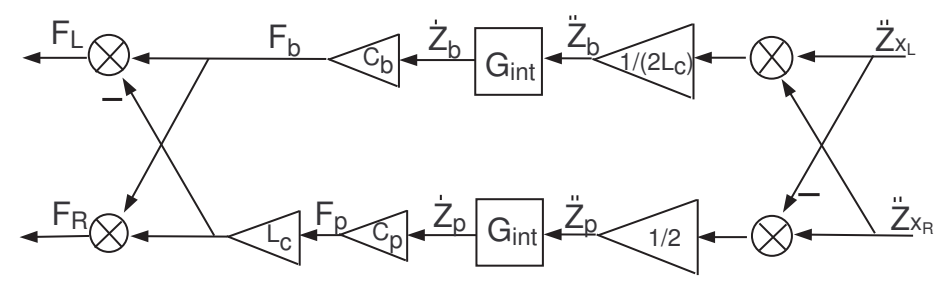

Figure 8: Modal control with skyhook damping 

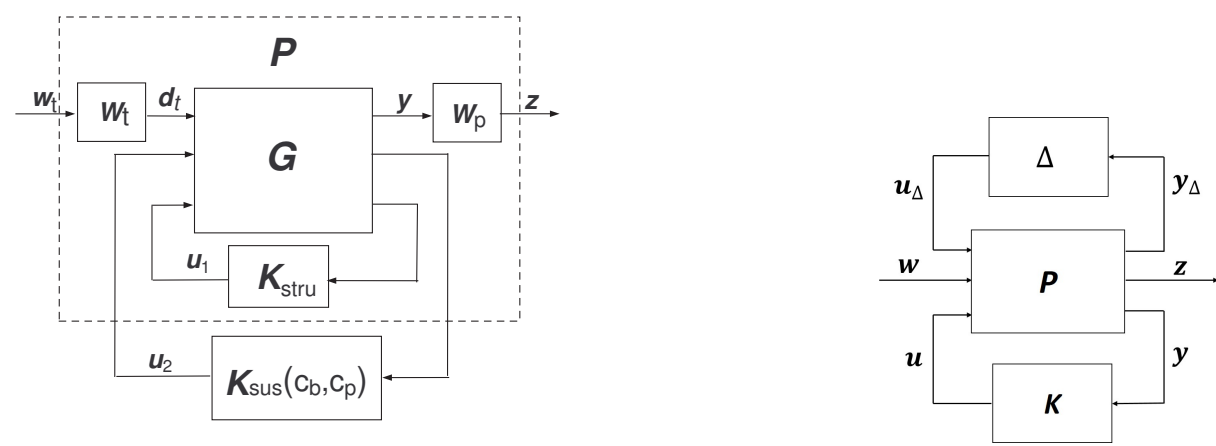

Figure 9: Design framework of controllerFigure 10: General structure for robust con$\boldsymbol{K}_{\text {sus }}$ trol and analysis

damper is dependent upon the absolute velocity of a vehicle body.

$$
\begin{aligned}
& F_{b}=-c_{b} \dot{Z}_{b} \\
& F_{c}=-c_{p} \dot{Z}_{p}
\end{aligned}
$$

where $\dot{Z}_{b}$ and $\dot{Z}_{p}$ are the absolute velocity of the bounce and pitch modes of the vehicle body and $c_{b}$ and $c_{p}$ are the modal skyhook damping gains, hence $F_{b}$ and $F_{c}$ are the modal control forces. In practice, the accelerations of the vehicle body are measured and then the signals are integrated to get the absolute velocities. A highpass filter is used along with an integrator in order to eliminate the long-term drift caused by the offset effect of the transducer due to environmental reasons. A second order Butterworth high-pass filter (HPF) is used

$$
H_{h p}=\frac{s^{2} / \omega^{2}}{s^{2} / \omega^{2}+s 2 \zeta / \omega+1}
$$

where the cut-off frequency $\omega$ is chosen to be significantly less than the main suspension frequency, and in this case is chosen to be $0.6 \mathrm{rad} / \mathrm{s}$.

\subsubsection{Structured $\mathcal{H}_{\infty}$ with skyhook damping}

The gains of the skyhook damper are tuned with fixed structured $\mathcal{H}_{\infty}$ synthesis, by which we are able to make the control efforts concentrate on the frequency range of concern without adding to the complexity of the resulting controller. Figure 9 shows the design scheme of the tuneable gains $c_{b}$ and $c_{p}$ of $\boldsymbol{K}_{\text {sus }}$ with structured $\mathcal{H}_{\infty}$, by which the design requirements are formulated as $\mathcal{H}_{\infty}$ constraints.

The generalised plant $\boldsymbol{P}$ includes the design model $\boldsymbol{G}$, which is the overall model with the second bending mode reduced, the sub-controller $\boldsymbol{K}_{\text {stru }}$ and the weighting matrices $\boldsymbol{W}_{t}$ and $\boldsymbol{W}_{\boldsymbol{p}} \cdot \boldsymbol{d}_{\boldsymbol{t}}$ is the track disturbance input with $\boldsymbol{W}_{t}$ reflects its spectrum, and $\boldsymbol{w}_{t}$ is the scaled track input vector. The regulated output $\boldsymbol{y}$ vector includes the accelerations at the centre and left and right suspension points of the vehicle body and the two control inputs, and the weighting matrix $\boldsymbol{W}_{\boldsymbol{p}}$ is specified according to the control requirements

$$
\boldsymbol{y}=\left[\ddot{Z}\left(x_{C}\right) \ddot{Z}\left(x_{L}\right) \ddot{Z}\left(x_{R}\right) F_{L} F_{R}\right]
$$




$$
\boldsymbol{W}_{\boldsymbol{p}}=\operatorname{diag}\left\{\left[W_{z} W_{z} W_{z} W_{u 2} W_{u 2}\right]\right\}
$$

where the dynamic weighting functions $W_{z}$ for the accelerations are chosen as a low pass-filters with corner frequencies $\omega_{z a}=50.3 \mathrm{rad} / \mathrm{s}$ and $\omega_{z b}=12.6 \mathrm{rad} / \mathrm{s}$ so as to suppress the vibrations of the rigid modes of low frequencies.

$$
W_{z}=k_{z} \frac{s^{2}+2 \zeta_{z} \omega_{z a} s+\omega_{z a}^{2}}{s^{2}+2 \zeta_{z} \omega_{z b} s+\omega_{z b}^{2}}
$$

The dynamic weighting function $W_{u 2}$ for control inputs are chosen as high-pass filters with corner frequencies $\omega_{u a}=12.6 \mathrm{rad} / \mathrm{s}$ and $\omega_{u b}=50.3 \mathrm{rad} / \mathrm{s}$ to constrain the control efforts within the lower frequency range, where the electro-hydraulic actuator has better performance.

$$
W_{u 2}=k_{u 2} \frac{s^{2}+2 \zeta_{u 2} \omega_{u a} s+\omega_{u a}^{2}}{s^{2}+2 \zeta_{u 2} \omega_{u b} s+\omega_{u b}^{2}}
$$

The coefficients $k_{z}$ and $k_{u 2}$ are tuned to achieve a good balance between performance and robustness.

The structured $\mathcal{H}_{\infty}$ synthesis requires solving semi-infinite, nonconvex, and nonsmooth optimisation of the form [33]

$$
\min _{C(s)}\left\|F_{l}\left(\boldsymbol{P}(s), \boldsymbol{K}_{\text {sus }}(s)\right)\right\|_{\infty}
$$

in which $F_{l}\left(\boldsymbol{P}(s), \boldsymbol{K}_{\text {sus }}(s)\right)$ is the transfer function from $\boldsymbol{w}_{t}$ to $\boldsymbol{z}$. Equation (59) is equivalent to

$$
\min _{c \in \mathbb{R}^{k}} \max _{\omega \in[0, \infty]} \bar{\sigma}\left(F_{l}(\widehat{\boldsymbol{P}}(j \omega), \boldsymbol{c})\right.
$$

where $\boldsymbol{c}$ gathers all low-level tunable parameters in $\boldsymbol{K}_{\text {sus }}(s)$, the two gains $c_{b}$ and $c_{p}$ , and the structure of $\boldsymbol{K}_{\text {sus }}$ is absorbed in $\widehat{P}$. The full details about the specialised nonsmooth optimisation techniques can be found in [34]

\section{Robustness analysis}

The stability robustness of the proposed controller is to be investigated via $\mu$ analysis technique. A brief background of $\mu$ analysis is provided in this section, and please refer to [32] for more details. $\mu$ analysis is based on the general structure for robust control design as shown in Figure 10. The uncertainties are pulled out of the system and collected in $\boldsymbol{\Delta}$ in block diagonal form

$$
\boldsymbol{\Delta}=\operatorname{diag}\left[\delta_{1} \boldsymbol{I}_{p 1}, \cdots, \delta_{k} \boldsymbol{I}_{p k}, \Delta_{a}, \Delta_{m}\right]
$$

while all the weighting functions are absorbed into $\boldsymbol{P}$, hence $\boldsymbol{P}$ is the generalised nominal plant. $\boldsymbol{\Delta}$ is connected with $\boldsymbol{P}$ via artificial inputs $\boldsymbol{u}_{\Delta}$ and outputs $\boldsymbol{y}_{\Delta} \cdot \boldsymbol{K}$ is the feedback controller connecting with $\boldsymbol{P}$ via the measured outputs $\boldsymbol{y}$ and control input $\boldsymbol{u}$ of the plant. $\boldsymbol{w}$ is the exogenous disturbance and $\boldsymbol{z}$ is the performance output.

For an uncertain system with uncertainty $\boldsymbol{\Delta}$, the structured singular value $\mu_{\Delta}$ is defined as the reciprocal of the smallest structured $\boldsymbol{\Delta}$ measured in terms of $\bar{\sigma}(\boldsymbol{\Delta})$ which makes the matrix $\boldsymbol{I}-\boldsymbol{M}_{11} \boldsymbol{\Delta}$ singular.

$$
\mu_{\Delta}\left(\boldsymbol{M}_{11}\right)=\frac{1}{\min _{\Delta} \bar{\sigma}(\boldsymbol{\Delta}) \mid \operatorname{det}\left(\boldsymbol{I}-\boldsymbol{M}_{11} \boldsymbol{\Delta}\right)=0}
$$


where $\boldsymbol{M}_{11}$ is the nominal closed-loop system from $\boldsymbol{u}_{\Delta}$ to $\boldsymbol{y}_{\Delta}$. The closed-loop system is robust stable against all considered uncertainties $\Delta$ if and only if $\mu_{\Delta}\left(\boldsymbol{M}_{11}\right)<1$, on the other hand the stability margin is defined as $\mu_{\Delta}^{-1}$ which provides an upper bound for the system's perturbation.

\section{Results and discussions}

The evaluation considers the system with model uncertainties, taking the overall model that considers the first two bending modes, extended with the dynamics of the electrohydraulic actuators with force tracking control [3], as the nominal model. Comparisons are made between the proposed decentralised controller $\boldsymbol{K}$ and the decentralised controller $\boldsymbol{K}_{1}$ [15], which consists of the sub-controller $\boldsymbol{K}_{\text {sus } 1}$ for active suspension control with skyhook damping tuned by trial and error, and the sub-controller $\boldsymbol{K}_{\text {stru } 1}$ for active structural damping with LQG control. $\boldsymbol{K}_{\text {stru1 }}$ and $\boldsymbol{K}_{\text {sus } 1}$ are designed in the same sequential manner with the same design model as for controllers $\boldsymbol{K}_{\text {stru }}$ and $\boldsymbol{K}_{\text {sus }}$ respectively as described in section 6 . The nominal vehicle parameters and the actuator and sensor specifications are given in the Appendices 1 and 2.

Firstly the nominal performance of the closed-loop system is analysed with the RMS values and PSDs of vehicle body accelerations and control inputs. Then performance robustness of two perturbed closed-loop systems within the bound of the given model uncertainties are analysed. Finally, the stability robustness of the uncertain closed-loop system is analysed with $\mu$ analysis technique against the model uncertainties.

Table 2 shows the RMS values of the accelerations at three performance positions (centre of the vehicle body and left and right suspension points), suspension deflections and control inputs in response to track irregularities for different controllers (including each sub-controller alone and the controller combining the two sub-controllers) with the nominal overall model that considers the first two bending modes, extended with the dynamics of the electro-hydraulic actuator with force tracking control. In general, controllers $\boldsymbol{K}_{\text {stru }}, \boldsymbol{K}_{\text {sus }}$ and $\boldsymbol{K}$ achieve slightly higher accelerations with less control efforts than $\boldsymbol{K}_{\text {stru } 1}, \boldsymbol{K}_{\text {sus } 1}$ and $\boldsymbol{K}_{1}$ respectively. It is understandable as improved robustness is expected at the cost of performance. Controller $\boldsymbol{K}_{\text {stru }}$ achieves substantial acceleration reduction at the centre position $x_{C}$, while the accelerations at $x_{L}$ and $x_{R}$ are only slightly reduced. This is because the centre acceleration is influenced the most by the first bending mode. Significant reductions in all three accelerations are achieved by controller $\boldsymbol{K}_{\text {sus }}$, while the centre acceleration is slightly higher than that with $\boldsymbol{K}_{\text {stru }}$. With combined controller $\boldsymbol{K}$, a further reduction in centre acceleration is brought by piezoelectric actuators compared to that with $\boldsymbol{K}_{\text {sus }}$. However, both the suspension control forces and piezoelectric actuator voltages are reduced compared to with $\boldsymbol{K}_{\text {stru }}$ or $\boldsymbol{K}_{\text {sus }}$ alone. This suggests that the combined approach helps to use the piezoelectric actuators and suspension actuators more efficiently to address the overall vibration issues.

Figure 11-13 compare the PSDs of accelerations at the three performance position$\mathrm{s}$ in response to track irregularities for different controllers with the nominal overall model. Figure 14 compares the PSDs of control inputs for active suspension and structural damping in different cases. The PSDs of the accelerations of the passive system prove that the first flexible mode (around $53 \mathrm{rad} / \mathrm{s}$ ) is the main cause of degradation 
Table 2: RMS results of the closed-loop systems with the nominal extended full model and different controllers

\begin{tabular}{|c|c|c|c|c|c|c|c|c|c|c|}
\hline \multirow{2}{*}{ Controller } & \multicolumn{3}{|c|}{$\begin{array}{c}\text { Vert. Acce. } \\
(\% \mathrm{~g})\end{array}$} & \multicolumn{2}{c|}{$\begin{array}{c}\text { Susp. Defl. } \\
(\mathrm{mm})\end{array}$} & \multicolumn{2}{c|}{$\begin{array}{c}\text { Susp. forces } \\
(\mathrm{kN})\end{array}$} & \multicolumn{3}{c|}{$\begin{array}{c}\text { Piezo Act.Voltages } \\
\text { (volts) }\end{array}$} \\
\cline { 2 - 12 } & centre & left & right & left & right & $F_{L}$ & $F_{R}$ & $U_{A_{1}}$ & $U_{A_{2}}$ & $U_{A_{3}}$ \\
\hline Passive & 3.04 & 3.04 & 3.75 & 7.8 & 11.35 & - & - & - & - & - \\
\hline $\boldsymbol{K}_{\text {stru }}$ & 1.89 & 2.75 & 3.80 & 7.81 & 11.36 & - & - & 150.03 & 150.02 & 150.02 \\
\hline $\boldsymbol{K}_{\text {sus }}$ & 2.19 & 2.25 & 2.34 & 8.53 & 9.88 & 2.46 & 2.80 & - & - & - \\
\hline $\boldsymbol{K}$ & 1.59 & 2.08 & 2.15 & 8.53 & 9.88 & 2.45 & 2.80 & 132.82 & 132.80 & 132.80 \\
\hline $\boldsymbol{K}_{\text {stru } 1}$ & 2.02 & 2.81 & 3.51 & 7.81 & 11.36 & - & - & 220.54 & 220.51 & 220.51 \\
\hline $\boldsymbol{K}_{\text {sus } 1}$ & 2.26 & 2.01 & 2.08 & 9.8 & 7.75 & 2.96 & 2.61 & - & - & - \\
\hline $\boldsymbol{K}_{1}$ & 1.45 & 1.76 & 1.74 & 9.8 & 7.75 & 2.95 & 2.6 & 196.42 & 196.4 & 196.4 \\
\hline
\end{tabular}

of ride quality while the second flexible mode (around $146 \mathrm{rad} / \mathrm{s}$ ) is as small as negligible. With structural damping controller alone, either $\boldsymbol{K}_{\text {stru }}$ or $\boldsymbol{K}_{\text {stru1 }}$, the first flexible mode vibration is effectively reduced. But the control effort from controller $\boldsymbol{K}_{\text {stru }}$ is less than $\boldsymbol{K}_{\text {stru1 }}$ and is more concentrated on the frequency range of the first flexible mode. With active suspension controller alone, either $\boldsymbol{K}_{\text {sus }}$ or $\boldsymbol{K}_{\text {sus } 1}$, the rigid mode vibrations at frequencies $<10 \mathrm{rad} / \mathrm{s}$ are substantially reduced while the first flexible mode vibration is only slightly reduced and the control effort from $\boldsymbol{K}_{\text {sus } 1}$ is slightly larger than $\boldsymbol{K}_{\text {sus }}$ with more peaks. With the combined structural damping and active suspension control, either $\boldsymbol{K}$ or $\boldsymbol{K}_{1}$, the performance is enhanced that both the rigid and flexible mode vibrations are effectively reduced, and less control efforts are demanded from each sub-controller and are more concentrated around their required frequencies ranges, which indicates the sub-controllers work more effectively in complementary to each other in the combined controllers. The control efforts demanded by $\boldsymbol{K}$ is in general less than controller $\boldsymbol{K}_{1}$, this also suggests that more efficient usage of the actuators is achieved with $\boldsymbol{K}$ than $\boldsymbol{K}_{1}$. It also shows that the controller $\boldsymbol{K}$ achieves lower level of accelerations at the centre position for frequencies $>113 \mathrm{rad} / \mathrm{s}$ than the controller $\boldsymbol{K}_{1}$, which means the $\boldsymbol{K}$ has less influence on high-frequency dynamics.

The performance robustness of the controllers are analysed with two perturbed model within the bound of uncertainties described in section 3. Figure 15(a) shows the max. /min. singular values of the passive system perturbed with increased passenger load, input multiplicative uncertainty and additive uncertainty, and Figure 15(b) shows the max. /min. singular values of the corresponding perturbed closed-loop systems with controller $\boldsymbol{K}_{1}$ and controller $\boldsymbol{K}$. Figure 16(a) shows the max. /min. singular values of the passive system perturbed with decreased stiffness, input multiplicative uncertainty and additive uncertainty, and Figure 16(b) shows the max. /min. singular values of the corresponding perturbed closed-loop systems with controller $\boldsymbol{K}_{1}$ and controller $\boldsymbol{K}$. It shows that both controllers are effective in reducing the targeted modes in the presence of the two perturbations. However, there is an increase of the singular values over high-frequency for controller $\boldsymbol{K}_{1}$ for both perturbations. This shows that controller $\boldsymbol{K}$ attains better performance robustness than controller $\boldsymbol{K}_{\mathbf{1}}$ in avoiding exciting high-frequency modes in the presence of high-frequency model uncertainties. 

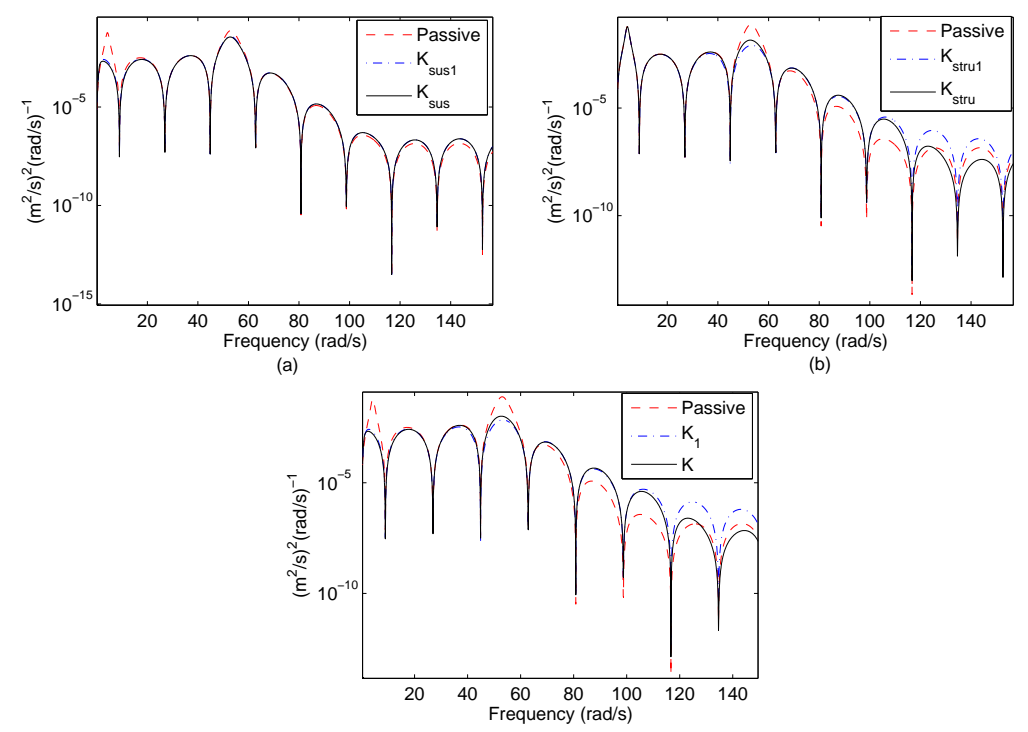

(c)

Figure 11: PSDs of accelerations at centre of the vehicle body: (a) with only suspension control (b) with only structural damping (c) with combined suspension control and structural damping
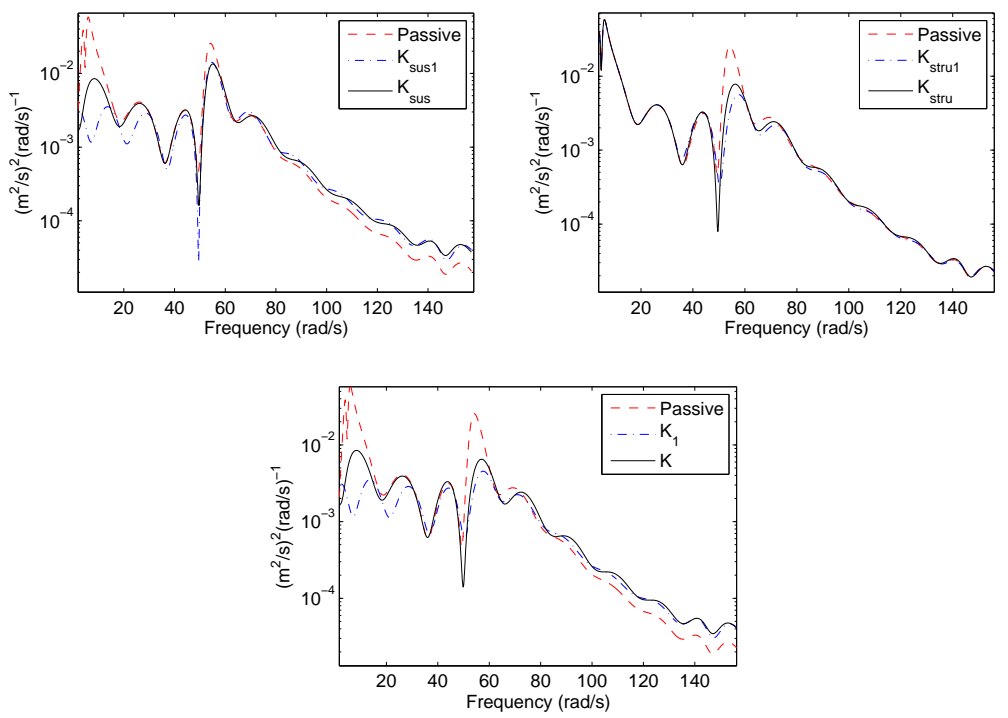

Figure 12: PSDs of accelerations at left suspension point of the vehicle body: (a) with only suspension control (b) with only structural damping (c) with combined suspension control and structural damping 

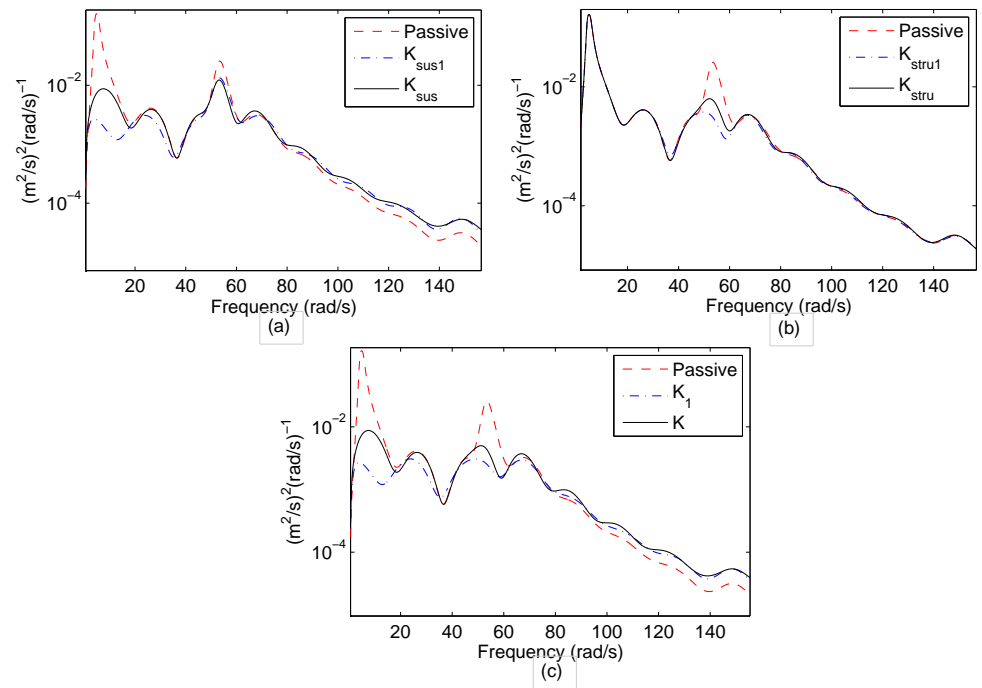

Figure 13: PSDs of accelerations at right suspension point of the vehicle body: (a) with only suspension control (b) with only structural damping (c) with combined suspension control and structural damping

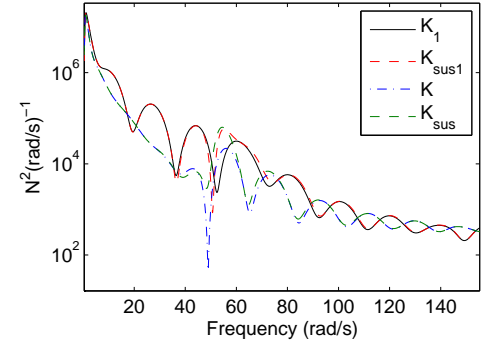

(a)

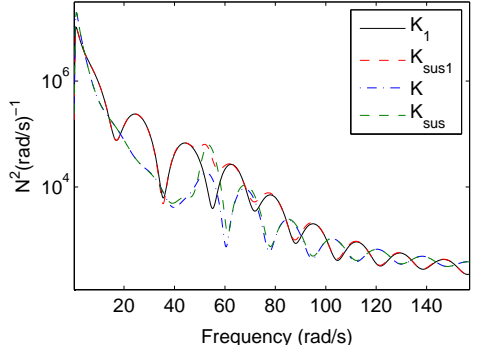

(b)

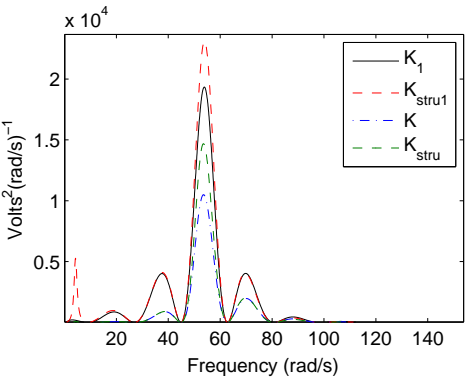

(c)

Figure 14: PSDs of control inputs: (a) left control force for active suspension (b) right force for active suspension (c) control inputs for structural damping 


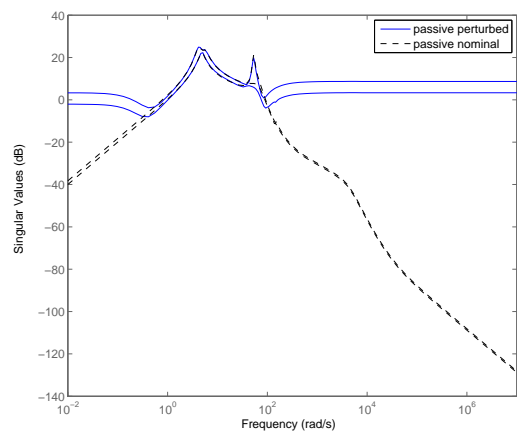

(a)

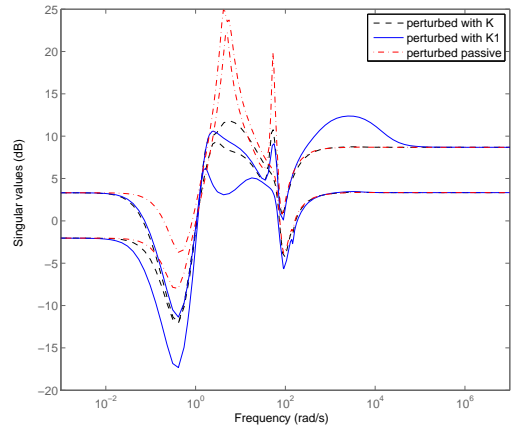

(b)

Figure 15: Max./min. singular values of (a) the model perturbed with increased passenger load, input multiplicative uncertainty and additive uncertainty (b) perturbed closed-loop systems with controller $K_{1}$ or controller $K$

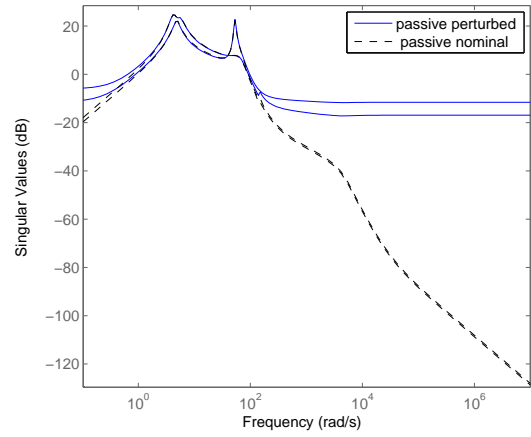

(a)

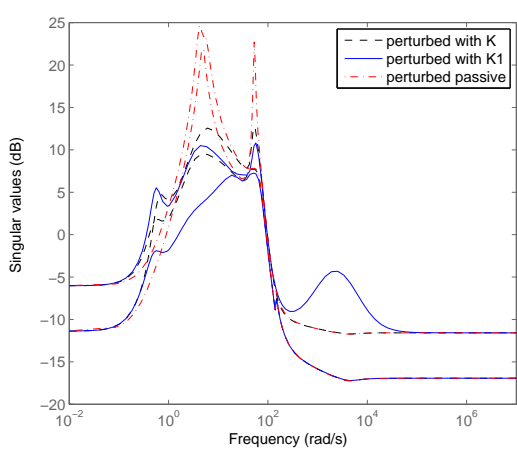

(b)

Figure 16: Max./min. singular values of (a) the model perturbed with decreased stiffness, input multiplicative uncertainty and additive uncertainty (b) perturbed closedloop systems with controller $K_{1}$ or controller $K$ 
Table 3: Lower bounds of the stability margins of the uncertain closed-loop systems

\begin{tabular}{|c|c|c|c|c|c|}
\hline Controller & Incre. Pass. load & Decre. Stiffness & Incre. Stiffness & Input Mult. Unc. & Add. Unc. \\
\hline $\boldsymbol{K}$ & 1.27 & 2.48 & 1.42 & 1.93 & 1.65 \\
\hline $\boldsymbol{K}_{1}$ & 1.27 & 2.56 & 1.42 & 1.40 & 0.65 \\
\hline
\end{tabular}

Stability robustness of the closed-loop system is evaluated using $\mu$-analysis techniques with respect to parametric uncertainties, input multiplicative uncertainties and additive uncertainties, as described in Section 3, separately. Table 3 summarises the lower bounds of the stability margins (defined as the reciprocal of the structured singular value). It shows that controller $\boldsymbol{K}$ and $\boldsymbol{K}_{1}$ achieve similar stability margins with respect to parametric uncertainties. Higher stability margins with respect to input multiplicative uncertainty and additive uncertainty are obtained by controller $\boldsymbol{K}$ than controller $\boldsymbol{K}_{1}$. With controller $\boldsymbol{K}$, the closed-loop system can bear up to 1.65 of the given level of additive uncertainty while controller $\boldsymbol{K}_{1}$ is not stable within the given level of additive uncertainty. This is because controller $\boldsymbol{K}$ is designed with frequency specification for the control inputs and regulated variables for each sub-controllers, making it less susceptible to input uncertainties and high-frequency model uncertainties.

Figure 17 compares the upper bounds of the structured singular values $\mu$ achieved by controller $\boldsymbol{K}$ with those achieved by controller $\boldsymbol{K}_{1}$ with respect to parametric uncertainties. It shows that controllers $\boldsymbol{K}$ and $\boldsymbol{K}_{1}$ achieve similar robustness against parametric uncertainties with the upper bound of $\mu$ being $<0.8$ for increased passenger load, $<0.4$ for decreased stiffness and $<0.71$ for increased stiffness. Figure 18 shows that the closed-loop system with controller $\boldsymbol{K}$ has lower values of $\mu$ than with controller $\boldsymbol{K}_{1}$ against input multiplicative uncertainty and additive uncertainty. It is noticed that with controller $\boldsymbol{K}_{1}, \mu$ increases and exceeds 1 over a high-frequency range as a result of the additive uncertainty, which indicates strong spill-over effects and instability may be caused by neglected high-frequency dynamics.

\section{Conclusions}

We have presented a decentralised control strategy combining active suspension control with skyhook damping and active structural damping with piezoelectric actuators and sensors for suppressing both the rigid and vertical bending mode of the railway vehicle to improve ride quality. The skyhook damping gains are tuned via structured $\mathcal{H}_{\infty}$ synthesis for controller robustness, while the structural damping controller is designed with frequency-dependent $\mathcal{H}_{2}$ control so that the piezoelectric actuators' effort concentrates on the frequency range of the targeted flexible mode to reduce spill-over effect. The design is based on a simplified side-view model of the railway vehicle considering only the rigid and first bending mode of the vehicle body. The placement of piezoelectric actuators and sensors is determined performance-wise with structural $\mathcal{H}_{2}$ norms. The evaluation of the controller uses the uncertain model considering parametric uncertainties, actuator uncertainties and uncertainties caused by neglected dynamics. It shows that the two sub-controllers work in complementary manner to each other that effective suppression of both the rigid and first bending mode vibration is achieved. Though 


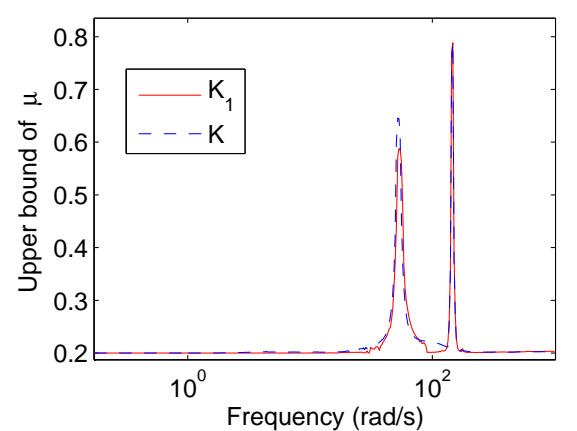

(a)

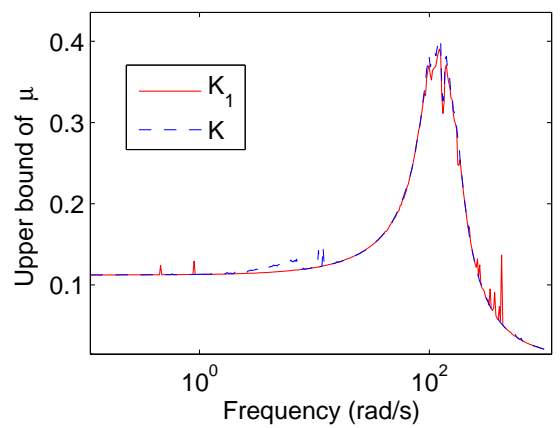

(b)

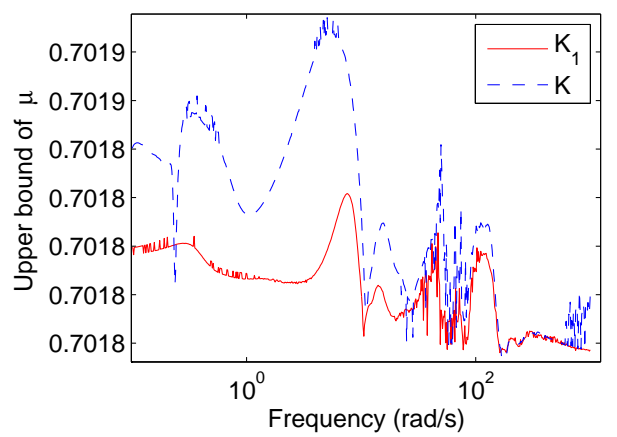

(c)

Figure 17: Upper bounds of $\mu$ for parametric uncertainties: (a) increased passenger load (b) decreased stiffness (c) increased stiffness

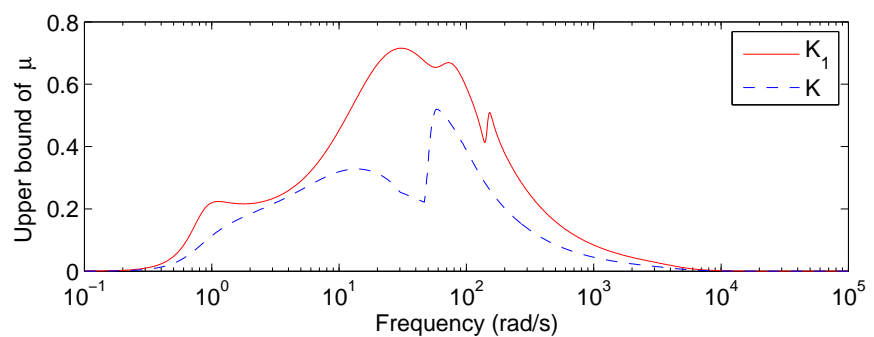

(a)

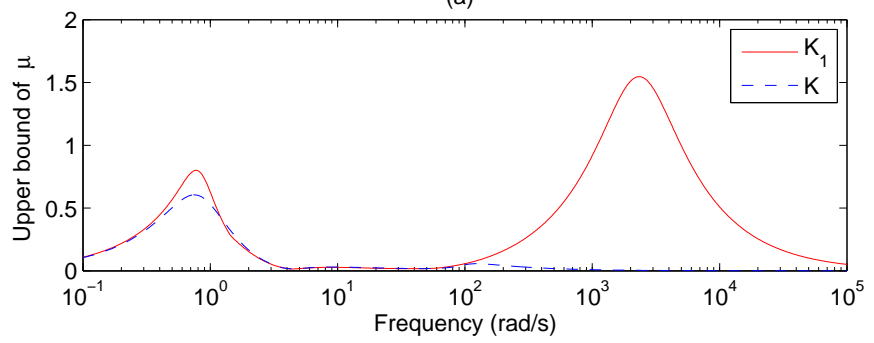

(b)

Figure 18: Upper bounds of $\mu$ for dynamic model uncertainties (a) input multiplicative uncertainty (b) additive uncertainty 
slightly higher accelerations are obtained with the proposed controller, in comparison with the more conventional controller combining LQG control and classical skyhook damping, the proposed controller is able to use the control efforts more efficiently and shows better performance robustness in avoiding spill-over effect in the presence of model uncertainties, and the robust $\mu$ analysis results show better stability robustness of the proposed controller with respect to input multiplicative uncertainty and additive uncertainty that account for neglected high-frequency dynamics. This proves the robustness of the proposed controller in achieving satisfactory reduction of the modes of concern while having little influence on the neglected high-frequency dynamics.

\section{References}

[1] Hac A, Youn I, Chen HH. Control of suspensions for vehicles with flexible bodiespart I: Active suspensions. J Dyn Syst Meas Control. 1996;118(3):509-517.

[2] Hac A, Youn I, Chen HH. Control of suspensions for vehicles with flexible bodiespart II: Semi-active suspensions. J Dyn Syst Meas Control. 1996;118(3):519-525.

[3] Zheng X. Active vibration control of flexible bodied railway vehilces via smart structures [dissertation]. Loughborough University; 2011.

[4] Md Yusof H, Goodall R, Dixon R. Control strategies for active secondary suspension actuators. In: Proceedings of the 22nd International Symposium on Dynamics of Vehicles on Roads and Tracks. Manchester, UK; 2011.

[5] Foo E, Goodall R. Active suspension control of flexible-bodied railway vehicles using electro-hydraulic and electro-magnetic actuators. Control Eng Pract. 2000; 8(5):507-518.

[6] Hansson J, Takano M, Takigami T, Tomioka, YSuzuki. Vibration suppression of railway vehicle carbody with piezoelectric elements (a study by using a scale model). JSME International Journal Series C. 2003;47(2):451-466.

[7] Takigami T, Tomioka T. Bending vibration suppression of railway vehicle carbody with piezoelectric elements(experimental results of excitation tests with a commuter car). Journal of Mechanical Systems for Transportation and Logistics. $2008 ; 1(1)$.

[8] Kamada T, Kiuchi R, Nagai M. Suppression of railway vehicle vibration by shunt damping using stack type piezoelectric transducers. Vehicle System Dynamics. 2008;46(Supplement):561-570.

[9] Kamada T, Tohtake T, Aiba T, Nagai M. Active vibration control of the railway vehicles by smart structure concept. Proceedings of International Symposium on Speed-up and Service Technology for Railway and Maglev Systems. 2005;

[10] Benatzky C, Kozek M, Jorgl HP. Comparison of controller design methods for a scaled metro vehicle - flexible structure experiments. In: Proceedings of the 2007 American Control Conference. New York, USA; 2007. p. 194-199. 
[11] Kozek M, Benatzky C, Schirrer A, Stribersky A. Vibration damping of a flexible car body structure using piezo-stack actuators . Control Eng Pract. 2011; 19(3):298-310.

[12] Schandl G, Lugner P, Benatzky C, Kozek M, Stribersky A. Comfort enhancement by an active vibration reduction system for a flexible railway car body. Vehicle System Dynamics. 2007;45(9):835-847.

[13] Schirrer A, Kozek M, Schoftner J. MIMO vibration control for a flexible rail car body: design and experimental validation. Vibration Analysis and Control - New Trends and Developments, InTech. 2011;.

[14] Kamada T, Hiraizumi K, Nagai M. Active vibration suppression of lightweight railway vehicle body by combined use of piezoelectric actuators and linear actuators. Vehicle System Dynamics. 2010;48(Supplement):73-87.

[15] Zheng X, Zolotas A, Goodall R. Combining active structural damping and active suspension control in flexible bodied railway vehicles. In: Proceedings of the 35th Chinese Control Conference. Chengdu, China; 2016. p. 8938-8944.

[16] Bruni S, Goodall R, Mei TX, Tsunashima H. Control and monitoring for railway vehicle dynamics. Vehicle System Dynamics. 2007;45(7-8):743-779.

[17] Piao M, Ren J, Li N, Zhao W. Comparative study on vertical vibration comfort of high-speed railway vehicle based on the suspension characteristics of air spring. China Railway Science. 2012;33(1):71-11.

[18] Benatzky C, Kozek M, Bilik C. Experimental control of a flexible beam using a stack-bending actuator principle. In: Proceedings of the 20th Scientific Conference. Hanoi,Vienam; 2006. p. 128-133.

[19] Halim D, Moheimani SR. Spatial resonant control of flexible structures - application to a piezoelectric laminate beam. IEEE transactions on control systems technology. 2001;9(1):37-53.

[20] Gevarter WB. Basic relations for control of flexible vehicles. AIAA Journal. 1970 April;8(4):666-672.

[21] Tomioka T, Takigami T. Experimental and numerical study on the effect due to passengers on flexural vibrations in railway vehicle carbodies. J Sound Vib. 2015; 343:1-19.

[22] Skogestad S, Postlethwaite I. Multivariable feedback control. Springer London; 2005.

[23] Schirrer A, Kozek M, Benatzky C. Piezo stack actuators in flexible structures: experimental verification of a nonlinear modeling and identification approach. In: Proceedings of Euromech Conference Enoc 2008. Saint Petersburg, Russia; 2008. 
[24] Tomioka T, Suzuki Y, Takigami T. Three-dimensional flexural vibration of lightweight railway vehicle carbody and a new analytical method for flexural vibration. Qr of Rtri. 2003;44(1):15-21.

[25] Stribersky A, Moser F, Rulka W. Structural dynamics and ride comfort of a rail vehicle system. Advances in Engineering Software. 2002;33:541-552.

[26] Iorga L, Baruh H, Ursu I. A review of $\mathcal{H}_{\infty}$ robust control of piezoelectric smart structures. Appl Mech Rev. 2008;61(4):040802-01-040802-15.

[27] Leleu S, Abou-Kandil H, Bonnasieux Y. Piezoelectric actuators and sensors location for active control of flexible structures. IEEE Trans Instrum Meas. 2001; 50(6):1577-1582.

[28] Gawronski WK. Advanced structural dynamics and active control of structures. Springer, New York; 1998.

[29] Moheimani S, TRyall. Considerations on placement of piezoceramic actuators that are used in structural vibration control. In: Proceedings of the Conference on Decision and Control. Arizona USA; 1999. p. 1118-1123.

[30] Pratt I. Active suspensions applied to railway trains [dissertation]. Loughborough University, UK; 1996.

[31] Dullerud GE, Paganini F. A course in robust control theory. Beijing world publishing corporation; 2014.

[32] Zhou K, Doyle JC, Glover K. Robust and optimal control. Englewood Cliffs: Prentice Hall; 1996.

[33] Gahinet P. Structured $\mathcal{H}_{\infty}$ synthesis in matlab. In: Proceedings of the 18 th World Congress, IFAC. Milano, Italy; 2011. p. 1435-1440.

[34] Apkarian P, Dominikus N. Nonsmooth $\mathcal{H}_{\infty}$ synthesis. IEEE Transactionis on Automatic Control. 2006;51(1):71-86.

\section{Appendix 1: Specifications of piezoelectric actuators and sensors}

Table 4: Specification of piezoelectric stack actuator ( PIEZOMECHANIK, Pst1000/35/200VS45 )

\begin{tabular}{ll}
\hline Maximum force & $50,000 \mathrm{~N}$ \\
Length, $L_{p x}$ & $0.194 \mathrm{~m}$ \\
Thickness of one disk & $5 \times 10^{-4} \mathrm{~m}$ \\
Diameter of disk & $0.045 \mathrm{~m}$ \\
Piezoelectric constant, $d_{33}$ & $6.80 \times 10^{-10} \mathrm{~m} /$ volts \\
Compliance, $s_{33}^{E}$ & $23 \times 10^{-12} \mathrm{~m}^{2} / \mathrm{N}$ \\
\hline
\end{tabular}


Table 5: Specification of piezoelectric sensor patch [19]

\begin{tabular}{ll}
\hline Length & $0.02 \mathrm{~m}$ \\
Thickness & $2.5 \times 10^{-4} \mathrm{~m}$ \\
Width & $0.025 \mathrm{~m}$ \\
Young's Modulus, $E_{p}$ & $6.70 \times 10^{10} \mathrm{~N} / \mathrm{m}^{2}$ \\
Capacitance, $C_{p}$ & $1.05 \times 10^{-7} \mathrm{~F}$ \\
Electromechanical coupling factor, $k_{31}$ & 0.34 \\
Voltage constant, $g_{31}$ & $-1.15 \times 10^{-2} \mathrm{Vm} / \mathrm{N}$ \\
\hline
\end{tabular}

\section{Appendix 2: Vehicle parameters}

Mass of the vehicle, $M_{v}$

Mass of the bogie, $M_{b}$

Center Mass, $M_{c}$

Body pitch Inertia, $I_{v c}$

Primary spring stiffness per axle, $K_{p}$

Primary damping per axle, $B_{p}$

Secondary spring stiffness per bogie, $K_{s}$

Secondary area stiffness per bogie, $K_{a}$

Secondary reservoir stiffness per bogie, $K_{s r}$

Secondary damping per bogie, $B_{s r}$

Centre spring, $K_{c}$

Centre damper, $B_{c}$

Bogie centre, $L_{b c}$

Vehicle+gangway, $L_{v}$

Speed of the train, $v$
$38000 \mathrm{~kg}$

$2500 \mathrm{~kg}$

$1000 \mathrm{~kg}$

$2.31 \times 10^{6} \mathrm{~kg} \mathrm{~m}^{2}$

$4.935 \mathrm{MN} / \mathrm{m}$

$0.05074 \mathrm{MNs} / \mathrm{m}$

$1.016 \mathrm{MN} / \mathrm{m}$

$0 \mathrm{~N} / \mathrm{m}$

$0.508 \mathrm{MN} / \mathrm{m}$

$0.06411 \mathrm{MN} / \mathrm{m}$

$39000 \mathrm{~N} / \mathrm{m}$

$628.32 \mathrm{Ns} / \mathrm{m}$

$19 \mathrm{~m}$

$27 \mathrm{~m}$

$198 \mathrm{~km} / \mathrm{h}$ 